\title{
SEMIGROUP-THEORETICAL CHARACTERIZATIONS OF ARITHMETICAL INVARIANTS WITH APPLICATIONS TO NUMERICAL MONOIDS AND KRULL MONOIDS
}

\author{
VÍCTOR BLANCO AND PEDRO A. GARCÍA-SÁNCHEZ AND ALFRED GEROLDINGER
}

\begin{abstract}
Arithmetical invariants - such as sets of lengths, catenary and tame degrees - describe the non-uniqueness of factorizations in atomic monoids. We study these arithmetical invariants by the monoid of relations and by presentations of the involved monoids. The abstract results will be applied to numerical monoids and to Krull monoids.
\end{abstract}

\section{INTRODUCTION}

Factorization theory describes the non-uniqueness of factorizations into irreducible elements of atomic monoids by arithmetical invariants, and it studies the relationship between these arithmetical invariants and algebraic invariants of the objects under consideration. Here, an atomic monoid means a commutative cancellative semigroup with unit element such that every non-unit may be written as a product of atoms (irreducible elements), and main examples are the multiplicative monoids consisting of the non-zero elements from a noetherian domain. In abstract semigroup theory, minimal relations and presentations are key tools to describe the algebraic structure of semigroups. Thus, there should be natural connections between the arithmetical invariants of factorization theory and the presentations of the semigroup. However, only first steps have been made so far to unveil these connections and to apply them successfully for further investigations. We mention two results in this direction (more can be found in the references). In [8], it was proved that the catenary degree of a monoid allows a description in terms of $\mathcal{R}$-equivalence classes (see Proposition 4.6 for details). In [34, semigroup-theoretical descriptions are used in the study of the arithmetic of non-principal orders in algebraic number fields.

The aim of the present paper is to explore further the connections between arithmetical invariants and semigroup-theoretical invariants, such as the monoid of relations and presentations. We discuss central invariants from factorization theory, such as the $\omega$-invariants (Section 3), the catenary and monotone catenary degrees (Section 4), the tame degrees (Section 5), and finally Section 6 deals with unions of sets of lengths. We provide - in the abstract setting of atomic monoids - new characterizations or new upper bounds (as in Propositions 3.3 5.2. Corollary 6.4), and reveal the influence of special presentations to the arithmetic (as in Theorem 5.6). Throughout, we apply the abstract results to concrete classes of monoids, mainly to numerical monoids and to Krull monoids (Corollaries 5.7 and 5.8. Theorem 6.6). Moreover, some of the results have relevance from the computational point of view, as they allow to provide explicit algorithms which partly have been implemented in GAP (see Example 3.6 or Remarks [5.9, and [13]). A more detailed discussion of the results will be given at the beginning of each section as soon as we have the required terminology at our disposal.

2010 Mathematics Subject Classification. 20M13, 20M14, 13A05.

Key words and phrases. presentations for semigroups, catenary degree, tame degree, sets of lengths, numerical monoid, Krull monoid.

This work was supported by the Ministerio de Educación y Ciencia (Project No. MTM2007-62346), by the Junta de Andalucia (Research Group FQM-343), and by the Austrian Science Fund FWF (Project No. P21576-N18). 


\section{Preliminaries}

We denote by $\mathbb{N}$ the set of positive integers, and we put $\mathbb{N}_{0}=\mathbb{N} \cup\{0\}$. For every $n \in \mathbb{N}$, we denote by $C_{n}$ a cyclic group with $n$ elements. For real numbers $a, b \in \mathbb{R}$, we set $[a, b]=\{x \in \mathbb{Z} \mid a \leq x \leq b\}$. Let $L, L^{\prime} \subset \mathbb{Z}$. We denote by $L+L^{\prime}=\left\{a+b \mid a \in L, b \in L^{\prime}\right\}$ their sumset. Two distinct elements $k, l \in L$ are called adjacent if $L \cap[\min \{k, l\}, \max \{k, l\}]=\{k, l\}$. A positive integer $d \in \mathbb{N}$ is called a distance of $L$ if there exist adjacent elements $k, l \in L$ with $d=|k-l|$. We denote by $\Delta(L)$ the set of distances of $L$. If $\emptyset \neq L \subset \mathbb{N}$, we call

$$
\rho(L)=\sup \left\{\frac{m}{n} \mid m, n \in L\right\}=\frac{\sup L}{\min L} \in \mathbb{Q}_{\geq 1} \cup\{\infty\}
$$

the elasticity of $L$, and we set $\rho(\{0\})=1$. By a monoid, we mean a commutative, cancellative semigroup with unit element.

\section{Throughout this paper, let $S$ be a monoid.}

We denote by $\mathcal{A}(S)$ the set of atoms (irreducible elements) of $S$, by $S^{\times}$the group of invertible elements, by $S_{\text {red }}=\left\{a S^{\times} \mid a \in S\right\}$ the associated reduced monoid of $S$, and by q $(S)$ a quotient group of $S$ with $S \subset \mathrm{q}(S)$. A submonoid $T \subset S$ is said to be saturated if $T=S \cap \mathrm{q}(T)$ (equivalently, if $a, b \in T$ and $a$ divides $b$ in $S$, then $a$ divides $b$ in $T$ ). We say that $S$ is reduced if $\left|S^{\times}\right|=1$. If not stated otherwise, we will use multiplicative notation. Submonoids of $\left(\mathbb{Z}^{s},+\right)$, in particular numerical monoids, will of course be written additively.

For a set $P$, we denote by $\mathcal{F}(P)$ the free (abelian) monoid with basis $P$. Then every $a \in \mathcal{F}(P)$ has a unique representation in the form

$$
a=\prod_{p \in P} p^{\mathrm{v}_{p}(a)} \quad \text { with } \quad \mathrm{v}_{p}(a) \in \mathbb{N}_{0} \text { and } \mathrm{v}_{p}(a)=0 \text { for almost all } p \in P .
$$

We call $|a|=\sum_{p \in P} \mathrm{v}_{p}(a)$ the length of $a$ and $\operatorname{supp}(a)=\left\{p \in P \mid \mathrm{v}_{p}(a)>0\right\} \subset P$ the support of $a$.

The free (abelian) monoid $\mathrm{Z}(S)=\mathcal{F}\left(\mathcal{A}\left(S_{\text {red }}\right)\right)$ is called the factorization monoid of $S$, the unique homomorphism

$$
\pi: \mathrm{Z}(S) \rightarrow S_{\text {red }} \quad \text { satisfying } \quad \pi(u)=u \quad \text { for each } \quad u \in \mathcal{A}\left(S_{\text {red }}\right)
$$

is called the factorization homomorphism of $S$ and

$$
\sim_{S}=\{(x, y) \in \mathrm{Z}(S) \times \mathrm{Z}(S) \mid \pi(x)=\pi(y)\}
$$

the monoid of relations of $S$. Clearly, we have $\mathrm{Z}(S) \cong\left(\mathbb{N}_{0}^{\left(\mathcal{A}\left(S_{\text {red }}\right)\right.},+\right)$, and if $S$ is written additively, then $\mathrm{Z}(S)$ and $\sim_{S}$ will be written additively too.

Let $\sigma \subset \sim_{S}$ be a subset. Then $\sigma^{-1}=\{(x, y) \in \sigma \mid(y, x) \in \sigma\}$, and $\sigma$ is called a presentation of $S$ if the congruence generated by $\sigma$ equals $\sim_{S}$ (equivalently, if $(x, y) \in \mathbf{Z}(S) \times \mathbf{Z}(S)$, then $(x, y) \in \sim_{S}$ if and only if there exist $z_{0}, \ldots, z_{k} \in \mathrm{Z}(S)$ such that $x=z_{0}, z_{k}=y$, and, for all $i \in[1, k],\left(z_{i-1}, z_{i}\right)=\left(x_{i-1} w_{i}, x_{i} w_{i}\right)$ with $w_{i} \in \mathbf{Z}(S)$ and $\left.\left(x_{i-1}, x_{i}\right) \in \sigma \cup \sigma^{-1}\right)$. A presentation $\sigma$ is said to be

- minimal if no proper subset of $\sigma$ generates $\sim_{S}$ (see [36, Chapter 9] for characterizations of minimal presentations in our setting).

- generic if $\sigma$ is minimal and for all $(x, y) \in \sigma$ we have $\operatorname{supp}(x y)=\mathcal{A}\left(S_{\text {red }}\right)$.

If $S$ has a generic presentation, then $S_{\text {red }}$ is finitely generated and has no primes.

For a subset $S^{\prime} \subset S$, we set $\mathrm{Z}\left(S^{\prime}\right)=\left\{z \in \mathrm{Z}(S) \mid \pi(z) \in S^{\prime}\right\}$. Let $Z \subset \mathrm{Z}(S)$ be a subset. We say that an element $x \in Z$ is minimal in $Z$ if for all elements $y \in Z$ with $y \mid x$ it follows that $x=y$. We denote by $\operatorname{Min}(Z)$ the set of minimal elements in $Z$. Let $x \in Z$. Since the number of elements $y \in Z$ with $y \mid x$ is finite, there exists an $x^{*} \in \operatorname{Min}(Z)$ with $x^{*} \mid x$. 
For $a \in S$, the set

$$
\begin{aligned}
& \mathrm{Z}(a)=\mathrm{Z}(\{a\}) \subset \mathrm{Z}(S) \text { is the set of factorizations of } a \text { and } \\
& \mathrm{L}(a)=\{|z| \mid z \in \mathbf{Z}(a)\} \subset \mathbb{N}_{0} \text { is the set of lengths of } a .
\end{aligned}
$$

By definition, we have $\mathrm{Z}(a)=\{1\}$ and $\mathrm{L}(a)=\{0\}$ for all $a \in S^{\times}$. The monoid $S$ is called atomic if $\mathrm{Z}(a) \neq \emptyset$ for all $a \in S$ (equivalently, every non-unit can be written as a product of atoms), and it is called factorial if $|\mathrm{Z}(a)|=1$ for all $a \in S$. If $S$ is reduced and atomic, then the set of atoms $\mathcal{A}(S)$ is the uniquely determined minimal generating set of $S$ ([25, Proposition 1.1.7]). We denote by $\mathcal{L}(S)=\{\mathrm{L}(a) \mid a \in S\}$ the system of sets of lengths of $S$, and by

$$
\Delta(S)=\bigcup_{L \in \mathcal{L}(S)} \Delta(L) \subset \mathbb{N}
$$

the set of distances of $S$.

For $z, z^{\prime} \in \mathrm{Z}(S)$, we can write

$$
z=u_{1} \cdot \ldots \cdot u_{l} v_{1} \cdot \ldots \cdot v_{m} \text { and } z^{\prime}=u_{1} \cdot \ldots \cdot u_{l} w_{1} \cdot \ldots \cdot w_{n},
$$

where $l, m, n \in \mathbb{N}_{0}$ and $u_{1}, \ldots, u_{l}, v_{1}, \ldots, v_{m}, w_{1}, \ldots, w_{n} \in \mathcal{A}\left(S_{\text {red }}\right)$ are such that

$$
\left\{v_{1}, \ldots, v_{m}\right\} \cap\left\{w_{1}, \ldots, w_{n}\right\}=\emptyset .
$$

Then $\operatorname{gcd}\left(z, z^{\prime}\right)=u_{1} \cdot \ldots \cdot u_{l}$, and we call

$$
\mathrm{d}\left(z, z^{\prime}\right)=\max \{m, n\}=\max \left\{\left|z \operatorname{gcd}\left(z, z^{\prime}\right)^{-1}\right|,\left|z^{\prime} \operatorname{gcd}\left(z, z^{\prime}\right)^{-1}\right|\right\} \in \mathbb{N}_{0}
$$

the distance between $z$ and $z^{\prime}$. For subsets $X, Y \subset \mathrm{Z}(S)$, we set

$$
\mathrm{d}(X, Y)=\min \{\mathrm{d}(x, y) \mid x \in X, y \in Y\} \in \mathbb{N}_{0},
$$

and thus $\mathrm{d}(X, Y)=0$ if and only if $(X \cap Y \neq \emptyset$ or $X=\emptyset$ or $Y=\emptyset)$.

Numerical Monoids. By a numerical monoid we mean a submonoid $S \subset\left(\mathbb{N}_{0},+\right)$ such that the complement $\mathbb{N} \backslash S$ is finite. The theory of numerical monoids is presented in the recent monograph [37. The connection to semigroup algebras and to one-dimensional local domains (in particular, to power series domains $K \llbracket S \rrbracket)$ is documented in the surveys [6, 4, 5] (all these are domains which have received a lot of attention in factorization theory). We shall make use of this in Section 5.

Let $S$ be a numerical monoid. Then $S$ is reduced and finitely generated. Suppose that $\mathcal{A}(S)=$ $\left\{n_{1}, \ldots, n_{t}\right\}$ with $t \in \mathbb{N}$ and $1<n_{1}<\ldots<n_{t}$. Then we write $S=\left\langle n_{1}, \ldots, n_{t}\right\rangle$, and since $\mathbb{N} \backslash S$ is finite, it follows that $\operatorname{gcd}\left(n_{1}, \ldots, n_{t}\right)=1$. Writing factorizations of an element $a \in S$ we put the atoms in boldface in order to distinguish between the atoms and the scalars. Thus $z=k_{1} \boldsymbol{n}_{\mathbf{1}}+\ldots+k_{t} \boldsymbol{n}_{\boldsymbol{t}}$, with $k_{1}, \ldots, k_{t} \in \mathbb{N}_{0}$, is the factorization of the element $a=\sum_{i=1}^{t} k_{i} n_{i} \in S$ of length $|z|=k_{1}+\ldots+k_{t}$. We denote by $\operatorname{Ap}(S, a)=\{s \in S \mid s-a \notin S\}$ the Apéry set of $a$ in $S$ (see [37]).

Krull monoids. The monoid $S$ is called a Krull monoid if it satisfies one of the following equivalent properties ([25, Theorem 2.4.8]):

(a) $S$ is $v$-noetherian and completely integrally closed,

(b) $S$ has a divisor theory,

(c) $S_{\text {red }}$ is a saturated submonoid of a free monoid.

The theory of Krull monoids is presented in the monographs [31, 30, 25]. Let $S$ be atomic. Clearly, $\sim_{S} \subset \mathrm{Z}(S) \times \mathrm{Z}(S)$ is saturated and hence $\sim_{S}$ is a Krull monoid by Property (c) (more on that can be found in [35, Lemma 11]), and hence, in particular, it is atomic. Moreover, if $S_{\text {red }}$ is finitely generated, then $\sim_{S}$ is finitely generated as a saturated submonoid of a finitely generated monoid (see 25, Proposition 2.7.5]). An integral domain $R$ is a Krull domain if and only if its multiplicative monoid $R \backslash\{0\}$ is a Krull monoid, and thus Property (a) shows that a noetherian domain is Krull if and only if it is integrally closed. 
Main portions of the arithmetic of a Krull monoid - in particular, all questions dealing with sets of lengths - can be studied in the associated monoid of zero-sum sequences over its class group. To provide this concept, let $G$ be an additive abelian group, $G_{0} \subset G$ a subset and $\mathcal{F}\left(G_{0}\right)$ the free monoid with basis $G_{0}$. According to the tradition of combinatorial number theory, the elements of $\mathcal{F}\left(G_{0}\right)$ are called sequences over $G_{0}$. For a sequence

$$
U=g_{1} \cdot \ldots \cdot g_{l}=\prod_{g \in G_{0}} g^{v_{g}(U)} \in \mathcal{F}\left(G_{0}\right),
$$

we call $\mathrm{v}_{g}(U)$ the multiplicity of $g$ in $U$,

$$
|U|=l=\sum_{g \in G} \mathrm{v}_{g}(U) \in \mathbb{N}_{0} \text { the length of } U, \text { and } \sigma(U)=\sum_{i=1}^{l} g_{i} \text { the sum of } U \text {. }
$$

The monoid

$$
\mathcal{B}\left(G_{0}\right)=\left\{U \in \mathcal{F}\left(G_{0}\right) \mid \sigma(U)=0\right\}
$$

is called the monoid of zero-sum sequences over $G_{0}$, and Property (c) shows that $\mathcal{B}\left(G_{0}\right)$ is a Krull monoid. We define the Davenport constant of $G_{0}$ by

$$
\mathrm{D}\left(G_{0}\right)=\sup \left\{|U| \mid U \in \mathcal{A}\left(\mathcal{B}\left(G_{0}\right)\right)\right\} \in \mathbb{N}_{0} \cup\{\infty\},
$$

which is a classical constant in Combinatorial Number Theory (see [18, 21).

We will use that for a reduced finitely generated monoid $S$ the following statements are equivalent ([25, Theorem 2.7.14]) :

- $S$ is a Krull monoid,

- $S$ is isomorphic to a monoid $\mathcal{B}\left(G_{0}\right)$ with $G_{0} \subset G$ as above,

- $S$ is isomorphic to a monoid of non-negative integer solutions of a system of linear Diophantine equations.

\section{THE $\omega$-INVARIANTS}

Definition 3.1. Let $S$ be atomic. For $b \in S$, let $\omega(S, b)$ denote the smallest $N \in \mathbb{N}_{0} \cup\{\infty\}$ with the following property:

For all $n \in \mathbb{N}$ and $a_{1}, \ldots, a_{n} \in S$, if $b \mid a_{1} \cdot \ldots \cdot a_{n}$, then there exists a subset $\Omega \subset[1, n]$ such that $|\Omega| \leq N$ and

$$
b \mid \prod_{\nu \in \Omega} a_{\nu}
$$

Furthermore, we set

$$
\omega(S)=\sup \{\omega(S, u) \mid u \in \mathcal{A}(S)\} \in \mathbb{N}_{0} \cup\{\infty\} .
$$

Let $S$ be atomic. By definition, an element $b \in S$ is a prime element if and only if $\omega(S, b)=1$, and $S$ is factorial if and only if $\omega(S)=1$. Thus these $\omega$-invariants (together with the associated tame degrees, see in particular Equation 5.2) measure in particular how far away atoms are from primes (see 28, 27, 26, 29]). An algorithm to compute the $\omega(S, \cdot)$ values in numerical monoids was recently presented in 3 . Here we start by showing that a slight variant of the property in the definition of $\omega(S, \cdot)$ does not change its value.

Lemma 3.2. Let $S$ be atomic and $b \in S$. Then $\omega(S, b)$ is the smallest $N \in \mathbb{N}_{0} \cup\{\infty\}$ with the following property: 
For all $n \in \mathbb{N}$ and $a_{1}, \ldots, a_{n} \in \mathcal{A}(S)$, if $b \mid a_{1} \cdot \ldots \cdot a_{n}$, then there exists a subset $\Omega \subset[1, n]$ such that $|\Omega| \leq N$ and

$$
b \mid \prod_{\nu \in \Omega} a_{\nu} .
$$

Proof. Let $\omega^{\prime}(S, b)$ denote the smallest integer $N \in \mathbb{N}_{0} \cup\{\infty\}$ satisfying the property mentioned in the lemma. We show that $\omega(S, b)=\omega^{\prime}(S, b)$. By definition, we have $\omega^{\prime}(S, b) \leq \omega(S, b)$. (Note, if $b \in S^{\times}$, then $\left.\omega(S, b)=\omega^{\prime}(S, b)=0\right)$.

In order to show that $\omega(S, b) \leq \omega^{\prime}(S, b)$, let $n \in \mathbb{N}$ and $a_{1}, \ldots, a_{n} \in S$ with $b \mid a_{1} \cdot \ldots \cdot a_{n}$. After renumbering if necessary there is an $m \in[0, n]$ such that $a_{1}, \ldots, a_{m} \in S \backslash S^{\times}$and $a_{m+1}, \ldots, a_{n} \in S^{\times}$. Then $b \mid a_{1} \cdot \ldots \cdot a_{m}$, and for every $i \in[1, m]$ we pick a factorization $a_{i}=u_{i, 1} \cdot \ldots \cdot u_{i, k_{i}}$ with $k_{i} \in \mathbb{N}$ and $u_{i_{1}}, \ldots, u_{i, k_{i}} \in \mathcal{A}(S)$. Then there is a subset $I \in[1, m]$ and, for every $i \in I$, a subset $\emptyset \neq \Lambda_{i} \subset\left[1, k_{i}\right]$ such that

which implies that $b \mid \prod_{i \in I} a_{i}$.

$$
|I| \leq \sum_{i \in I}\left|\Lambda_{i}\right| \leq \omega^{\prime}(S, b) \quad \text { and } \quad b \mid \prod_{i \in I} \prod_{\nu \in \Lambda_{i}} u_{i, \nu}
$$

The forthcoming characterization of $\omega(S)$ will be easy to prove. But it is useful from a computational point of view, as well as it will be a key ingredient in the proof of Theorem 5.6 .

Proposition 3.3. Let $S$ be atomic.

1. For every $s \in S$ we have

$$
\omega(S, s)=\sup \{|x| \mid x \in \operatorname{Min}(\mathbf{Z}(s S))\} .
$$

2. $\omega(S)=\sup \{|x| \mid x \in \operatorname{Min}(\mathbf{Z}(u S))$ for some $u \in \mathcal{A}(S)\}$.

Proof. Obviously, it is sufficient to prove the first statement. Furthermore, we may assume that $S$ is reduced.

Let $x=\prod_{u \in \mathcal{A}(S)} u^{m_{u}} \in \operatorname{Min}(\mathrm{Z}(s S))$. Since $x \in \mathbf{Z}(s S)$, it follows that $s$ divides $\prod_{u \in \mathcal{A}(S)} u^{m_{u}}$ (in $S$ ), and since $x$ is minimal in $\mathrm{Z}(s S), s$ does not divide a proper subproduct. Therefore, we get that $\omega(S, s) \geq \sum_{u \in \mathcal{A}(S)} m_{u}=|x|$.

Conversely, let $\left(m_{u}\right)_{u \in \mathcal{A}(S)} \in \mathbb{N}_{0}^{\mathcal{A}(S)}$ be such that $s$ divides $\prod_{u \in \mathcal{A}(S)} u^{m_{u}}$ (in $\left.S\right)$. Then $x=\prod_{u \in \mathcal{A}(S)} u^{m_{u}} \in$

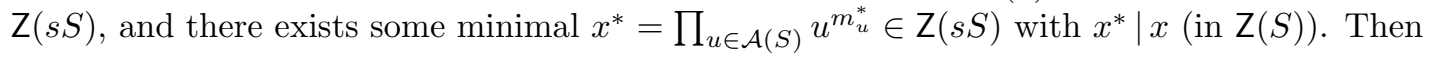

$$
s\left|\prod_{u \in \mathcal{A}(S)} u^{m_{u}^{*}}\right| \prod_{u \in \mathcal{A}(S)} u^{m_{u}} \quad(\text { in } S)
$$

and hence $\omega(S, s) \leq \sum_{u \in \mathcal{A}(S)} m_{u}^{*} \leq \sup \{|y| \mid y \in \operatorname{Min}(\mathrm{Z}(s S))\}$.

\section{Remarks 3.4.}

1. Note that $\omega(S, s)$ is finite for all $s \in S$ not only for finitely generated monoids, but more generally for all $v$-noetherian monoids (see [27, Theorem 4.2]).

2. Let $k, r \in \mathbb{N}_{0}$ and $n, d_{1}, \ldots, d_{k} \in \mathbb{N}$. Let $S \subset\left(\mathbb{N}_{0}^{n},+\right)$ be the set of all non-negative integer solutions $\left(x_{1}, \ldots, x_{n}\right) \in \mathbb{N}_{0}^{n}$ of the following system of equations

$$
\begin{array}{ll}
a_{1,1} x_{1}+\cdots+a_{1, n} x_{n} & \equiv 0 \bmod d_{1}, \\
a_{k, 1} x_{1}+\cdots+a_{k, n} x_{n} & \equiv 0 \bmod d_{k}, \\
a_{k+1,1} x_{1}+\cdots+a_{k+1, n} x_{n} & =0, \\
& \vdots \\
a_{k+r, 1} x_{1}+\cdots+a_{k+r, n} x_{n} & =0
\end{array}
$$


where all $a_{i, j}$ are integers. Obviously, $S \subset\left(\mathbb{N}_{0}^{n},+\right)$ is a submonoid with

$$
\mathrm{q}(S) \cap \mathbb{N}_{0}^{n}=S .
$$

Let $G=\mathbb{Z} / d_{1} \mathbb{Z} \times \ldots \times \mathbb{Z} / d_{k} \mathbb{Z} \times \mathbb{Z}^{r}$ and

$$
G_{0}=\left\{\left(a_{1, i}+d_{1} \mathbb{Z}, \ldots, a_{k, i}+d_{k} \mathbb{Z}, a_{k+1, i}, \ldots, a_{k+r, i}\right) \in G \mid i \in[1, n]\right\} .
$$

Then $S$ is obviously isomorphic to $\mathcal{B}\left(G_{0}\right)$, the monoid of zero-sum sequences over $G_{0}$. This (or independently, the fact that $S \subset\left(\mathbb{N}_{0}^{n},+\right)$ is saturated) show that $S$ is a reduced, finitely generated Krull monoid. If a finitely generated Krull monoid is given in that form, then the characterization of Proposition 3.3 turns out to be extremely useful, as the next corollary illustrates.

Corollary 3.5. Let $S \subset\left(\mathbb{N}_{0}^{n},+\right)$ be a saturated submonoid with $\mathcal{A}(S)=\left\{\boldsymbol{s}_{\mathbf{1}}, \ldots, \boldsymbol{s}_{\boldsymbol{t}}\right\}$, where $n, t \in \mathbb{N}$, and let $A \in M_{n, t}(\mathbb{Z})$ be the matrix whose columns are $s_{1}, \ldots, s_{t}$. If $s \in S$ and $\left(x_{1}, \ldots, x_{t}\right) \in \mathbb{N}_{0}^{t}$, then

$$
x_{1} s_{\mathbf{1}}+\ldots+x_{t} \boldsymbol{s}_{\boldsymbol{t}} \in \mathrm{Z}(s+S) \quad \text { if and only if } A\left(\begin{array}{c}
x_{1} \\
\vdots \\
x_{t}
\end{array}\right) \geq s .
$$

Proof. Observe that, by definition of $A$, we have for $s$ and $\left(x_{1}, \ldots, x_{t}\right)$ as above, that

$$
x_{1} s_{\mathbf{1}}+\ldots+x_{t} \boldsymbol{s}_{\boldsymbol{t}} \in \mathrm{Z}(s) \quad \text { if and only if } A\left(\begin{array}{c}
x_{1} \\
\vdots \\
x_{t}
\end{array}\right)=s .
$$

If $x_{1} s_{1}+\ldots+x_{t} s_{\boldsymbol{t}} \in \mathrm{Z}(s+S)$, then there is some $s^{\prime} \in S$ such that $x_{1} s_{\mathbf{1}}+\ldots+x_{t} s_{\boldsymbol{t}}=s+s^{\prime} \in S$, and hence $A\left(x_{1}, \ldots, x_{t}\right)^{t}=s+s^{\prime} \geq s$.

Conversely, let $x \in \mathbb{N}_{0}^{t}$ (considered as a column) be such that $A x \geq s$. Then $s^{\prime}=A x \in S$ and $s^{\prime}-s \in \mathrm{q}(S) \cap \mathbb{N}_{0}^{n}=S$. Thus $s^{\prime} \in s+S$ and $x_{1} s_{1}+\ldots+x_{t} s_{\boldsymbol{t}} \in \mathrm{Z}\left(s^{\prime}\right) \subset \mathrm{Z}(s+S)$.

The following Example 3.6 illustrates how Proposition 3.3 and Corollary 3.5 can be used to calculate the $\omega$-invariants. It was performed by using an algorithm due to E. Contejean and H. Devie with slack variables (see [12]; these authors published later a paper to avoid the use of these extra variables).

Example 3.6. Let $S \subset\left(\mathbb{N}_{0}^{3},+\right)$ be the set of non-negative integer solutions of

$$
\begin{aligned}
& x+z=0 \quad \bmod 2 \\
& y+z=0 \quad \bmod 2 .
\end{aligned}
$$

Then

$$
\mathcal{A}(S)=\left\{\left(\begin{array}{l}
2 \\
0 \\
0
\end{array}\right),\left(\begin{array}{l}
0 \\
2 \\
0
\end{array}\right),\left(\begin{array}{l}
0 \\
0 \\
2
\end{array}\right),\left(\begin{array}{l}
1 \\
1 \\
1
\end{array}\right)\right\},
$$

and $S$ is isomorphic to $\mathcal{B}\left(G_{0}\right)$ with $G_{0}=(\mathbb{Z} / 2 \mathbb{Z} \times \mathbb{Z} / 2 \mathbb{Z}) \backslash\{(0,0)\}$. The set of solutions of

$$
\left(\begin{array}{llll}
2 & 0 & 0 & 1 \\
0 & 2 & 0 & 1 \\
0 & 0 & 2 & 1
\end{array}\right) x \geq\left(\begin{array}{l}
1 \\
1 \\
1
\end{array}\right)
$$

is $\{(0,0,0,1),(1,1,1,0)\}+\mathbb{N}_{0}^{4}$, and thus, by Proposition 3.3 and Corollary $3.5, \omega(S,(1,1,1))=3$. The set of solutions of

$$
\left(\begin{array}{llll}
2 & 0 & 0 & 1 \\
0 & 2 & 0 & 1 \\
0 & 0 & 2 & 1
\end{array}\right) x \geq\left(\begin{array}{l}
2 \\
0 \\
0
\end{array}\right)
$$


is $\{(1,0,0,0),(0,0,0,2)\}+\mathbb{N}_{0}^{4}$, whence $\omega(S,(2,0,0))=2$. By symmetry, we get $\omega(S,(0,2,0))=2=$ $\omega(S,(0,0,2))$. Thus it follows that $\omega(S)=3$.

\section{The CATENARY And MONOtONE CATENARY DEGREeS}

Definition 4.1. Let $S$ be atomic and $a \in S$.

1. Let $z, z^{\prime} \in \mathrm{Z}(a)$ be factorizations of $a$ and $N \in \mathbb{N}_{0} \cup\{\infty\}$. A finite sequence $z_{0}, z_{1}, \ldots, z_{k}$ in $Z(a)$ is called an $N$-chain of factorizations from $z$ to $z^{\prime}$ if $z=z_{0}, z^{\prime}=z_{k}$ and $\mathrm{d}\left(z_{i-1}, z_{i}\right) \leq N$ for every $i \in[1, k]$. In addition, the chain is called monotone if $\left|z_{0}\right| \leq \ldots \leq\left|z_{k}\right|$ or $\left|z_{0}\right| \geq \ldots \geq\left|z_{k}\right|$.

If there exists a (monotone) $N$-chain of factorizations from $z$ to $z^{\prime}$, we say that $z$ and $z^{\prime}$ can be concatenated by a (monotone) $N$-chain.

2. We denote by $\mathrm{c}(a) \in \mathbb{N}_{0} \cup\{\infty\}$ (or by $\mathrm{c}_{\text {mon }}(a)$ resp.) the smallest $N \in \mathbb{N}_{0} \cup\{\infty\}$ such that any two factorizations $z, z^{\prime} \in \mathrm{Z}(a)$ can be concatenated by an $N$-chain (or by a monotone $N$-chain).

3. Moreover,

$\mathrm{c}(S)=\sup \{\mathrm{c}(b) \mid b \in S\} \in \mathbb{N}_{0} \cup\{\infty\} \quad$ and $\quad \mathrm{c}_{\text {mon }}(S)=\sup \left\{\mathrm{c}_{\text {mon }}(b) \mid b \in S\right\} \in \mathbb{N}_{0} \cup\{\infty\}$

denote the catenary degree and the monotone catenary degree of $S$.

Whereas the catenary degree is a classic invariant in factorization theory, the monotone catenary degree was introduced only in [14]. However, since then the existence of monotone and of near monotone chains of factorizations have been investigated in various aspects (see [15, 16, 23]). The monotone catenary degree is always (explicitly or implicitly) studied in a two-step procedure.

Definition 4.2. Let $S$ be atomic and $a \in S$.

1. For $k \in \mathbb{Z}$, let $\mathbf{Z}_{k}(a)=\{z \in \mathbf{Z}(a)|| z \mid=k\}$ denote the set of factorizations of $a$ having length $k$. We define

and we set

$$
\mathrm{c}_{\text {adj }}(a)=\sup \left\{\mathrm{d}\left(\mathrm{Z}_{k}(a), \mathrm{Z}_{l}(a)\right) \mid k, l \in \mathrm{L}(a) \text { are adjacent }\right\}
$$

$$
\mathrm{c}_{\mathrm{adj}}(S)=\sup \left\{\mathrm{c}_{\mathrm{adj}}(b) \mid b \in S\right\} \in \mathbb{N}_{0} \cup\{\infty\} .
$$

2. Let $\mathrm{C}_{\text {equal }}(a)$ denote the smallest $N \in \mathbb{N}_{0} \cup\{\infty\}$ with the following property:

For all $z, z^{\prime} \in \mathrm{Z}(a)$ with $|z|=\left|z^{\prime}\right|$ there exists a monotone $N$-chain concatenating $z$ and $z^{\prime}$. We call

$$
\mathrm{c}_{\text {equal }}(S)=\sup \left\{\mathrm{c}_{\text {equal }}(b) \mid b \in S\right\} \in \mathbb{N}_{0} \cup\{\infty\}
$$

the equal catenary degree of $S$.

Obviously, we have

$$
\mathrm{c}(a) \leq \mathrm{c}_{\text {mon }}(a)=\sup \left\{\mathrm{c}_{\text {equal }}(a), \mathrm{c}_{\text {adj }}(a)\right\} \leq \sup \mathrm{L}(a) \quad \text { for all } \quad a \in S,
$$

and hence

$$
\mathrm{c}(S) \leq \mathrm{c}_{\text {mon }}(S)=\sup \left\{\mathrm{c}_{\text {equal }}(S), \mathrm{c}_{\text {adj }}(S)\right\} .
$$

It is well-known that the monotone catenary degree $c_{\text {mon }}(S)$ is finite for finitely generated monoids ([14, Theorem 3.9]), and hence also for Krull monoids with finite class group, because it is stable under transfer homomorphisms. Our results will provide a more natural upper bound for $\mathrm{c}_{\mathrm{mon}}(S)$, valid among others finitely generated monoids. Inequality 5.1] will show that there is a canonical chain of inequalities involving the set of distances, the $\omega$-invariants, and the catenary and tame degrees. However, there seems to be no obvious relationship between $c_{\text {mon }}(\cdot)$, on the one side and $\omega(\cdot)$, the tame degree or on their canonical 
upper bound (see Proposition 5.2) on the other side. We study these phenomena by investigating $\mathrm{c}_{\text {equal }}(\cdot)$, and $\mathrm{c}_{\mathrm{adj}}(\cdot)$ individually, and summarize our discussion after Proposition 5.2

Definition 4.3. Let $S$ be atomic. Then

$$
\sim_{S, \text { equal }}=\{(x, y) \in \mathbf{Z}(S) \times \mathbf{Z}(S) \mid \pi(x)=\pi(y) \text { and }|x|=|y|\}
$$

is called the monoid of equal-length relations of $S$.

Proposition 4.4. Let $S$ be atomic.

1. $\sim_{S \text {,equal }} \subset \sim_{S}$ is a saturated submonoid, and hence $\sim_{S \text {,equal }}$ is a Krull monoid.

2. If $S_{\text {red }}$ is finitely generated, then $\sim_{S \text {,equal }}$ is finitely generated.

3. $\mathrm{C}_{\text {equal }}(S) \leq \sup \left\{|x| \mid(x, y) \in \mathcal{A}\left(\sim_{S \text {, equal }}\right)\right.$ for some $\left.y \in \mathrm{Z}(S)\right\}$.

4. For $d \in \Delta(S)$ let $A_{d}=\{x \in \mathrm{Z}(S)|d+| x \mid \in \mathrm{L}(\pi(x))\}$. Then $\mathrm{c}_{\text {adj }}(S) \leq \sup \left\{d+|x| \mid x \in \operatorname{Min}\left(A_{d}\right), d \in \Delta(S)\right\}$.

Proof. 1. Obviously, $\sim_{S \text {,equal }}$ is a submonoid of $\sim_{S}$. In order to show that it is saturated, let $\left(x_{1}, x_{2}\right),\left(z_{1}, z_{2}\right)$ $\in \sim_{S \text {,equal }}$ be such that $\left(x_{1}, x_{2}\right)$ divides $\left(z_{1}, z_{2}\right)$ in $\sim_{S}$. Then there exists $\left(y_{1}, y_{2}\right) \in \sim_{S}$ such that $x_{1} y_{1}=z_{1}$ and $x_{2} y_{2}=z_{2}$. This implies that $\left|y_{1}\right|=\left|z_{1}\right|-\left|x_{1}\right|=\left|z_{2}\right|-\left|x_{2}\right|=\left|y_{2}\right|$, and hence $\left(y_{1}, y_{2}\right) \in \sim_{S \text {,equal }}$. Thus $\sim_{S \text {,equal }} \subset \sim_{S}$ is a saturated. Since $\sim_{S}$ is a Krull monoid by [35, Lemma 11], $\sim_{S}$, equal is a Krull monoid by [25, Proposition 2.4.4].

2. Let $S_{\text {red }}$ be finitely generated. Then $\sim_{S}$ is finitely generated (as observed in Section 2), and hence $\sim_{S \text {,equal }}$ is finitely generated as a saturated submonoid of a finitely generated monoid (25), Proposition 2.7.5]).

3. We set $M=\sup \left\{|x| \mid(x, y) \in \mathcal{A}\left(\sim_{S \text {,equal }}\right)\right.$ for some $\left.y \in \mathrm{Z}(S)\right\}$, and have to show that $\mathrm{c}_{\text {equal }}(a) \leq M$

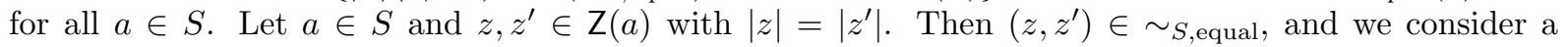
factorization, say

$$
\left(z, z^{\prime}\right)=\left(x_{1}, x_{1}^{\prime}\right) \cdot \ldots \cdot\left(x_{k}, x_{k}^{\prime}\right) \quad \text { where } \quad\left(x_{i}, x_{i}^{\prime}\right) \in \mathcal{A}\left(\sim_{S, \text { equal }}\right) \text { for all } i \in[1, k] .
$$

Then

$$
z=z_{0}, z_{1}=x_{1}^{\prime} x_{2} \cdot \ldots \cdot x_{k}, \ldots, z_{i}=x_{1}^{\prime} \cdot \ldots \cdot x_{i}^{\prime} x_{i+1} \cdot \ldots \cdot x_{k}, \ldots, z_{k}=x_{1}^{\prime} \cdot \ldots \cdot x_{k}^{\prime}=z^{\prime}
$$

is an $M$-chain of factorizations from $z$ to $z^{\prime}$ and with $\left|z_{i}\right|=|z|$ for all $i \in[0, k]$.

4. Let $a \in S$ and $k, l \in \mathrm{L}(a)$ be adjacent lengths, say $l-k=d \in \Delta(S)$. We pick some $z \in \mathrm{Z}_{k}(a)$. Then there exists some $x \in \operatorname{Min}\left(A_{d}\right)$ such that $x \mid z$. If $x^{\prime} \in \mathbf{Z}(\pi(x))$ with $\left|x^{\prime}\right|-|x|=d$, then $z^{\prime}=x^{\prime}\left(x^{-1} z\right) \in$ $\mathrm{Z}_{l}(a)$ and $\mathrm{d}\left(\mathrm{Z}_{k}(a), \mathrm{Z}_{l}(a)\right) \leq \mathrm{d}\left(z, z^{\prime}\right) \leq|x|+d$. This shows that $\mathrm{c}_{\text {adj }}(a) \leq \sup \left\{d+|x| \mid x \in \operatorname{Min}\left(A_{d}\right), d \in\right.$ $\Delta(S)\}$, and hence the assertion follows.

Proposition 4.4 2 will allow us to obtain a more explicit finiteness criterion for $\mathrm{c}_{\mathrm{adj}}(S)$ in Proposition 5.2

Definition 4.5. Let $S$ be atomic.

1. Two elements $z, z^{\prime} \in \mathrm{Z}(S)$ are $\mathcal{R}$-related if $z=z^{\prime}=1$ or if $z$ and $z^{\prime}$ can be concatenated by a chain of factorizations $z=z_{0}, \ldots, z_{k}=z^{\prime}$ such that $\pi\left(z_{i}\right)=\pi(z)$ and $\operatorname{gcd}\left(z_{i-1}, z_{i}\right) \neq 1$ for all $i \in[1, k]$.

2. For $a \in S$, we denote by $\mathcal{R}_{a}$ the set of $\mathcal{R}$-(equivalence) classes of $\mathrm{Z}(a)$. For $\sigma \in \mathcal{R}_{a}$ we set $|\sigma|=\min \{|z| \mid z \in \sigma\}$, and we define

$$
\mu(a)=\sup \left\{|\sigma| \mid \sigma \in \mathcal{R}_{a}\right\} .
$$

3. We set

$$
\mu(S)=\sup \left\{\mu(a) \mid a \in S \text { with }\left|\mathcal{R}_{a}\right| \geq 2\right\}
$$


We will need the following result, first proved for finitely generated monoids (see [8, Theorem 3.1]) and then in the general setting (see [35, Corollary 9]).

Proposition 4.6. If $S$ is atomic, then $\mathrm{c}(S)=\mu(S)$.

There is no analogue result for the monotone catenary degree. Below we will provide the first example of a monoid which is not tame but which has finite monotone catenary degree (indeed the catenary and the monotone catenary degree coincide). On the other side of the spectrum there are tame monoids with infinite monotone catenary degree. We recall the notion of finitely primary monoids, a concept which stems from ring theory. The monoid $S$ is called finitely primary if there exist $s, \alpha \in \mathbb{N}$ with the following properties:

$S$ is a submonoid of a factorial monoid $F=F^{\times} \times\left[p_{1}, \ldots, p_{s}\right]$ with $s$ pairwise non-associated prime elements $p_{1}, \ldots, p_{s}$ satisfying

$$
S \backslash S^{\times} \subset p_{1} \cdot \ldots \cdot p_{s} F \text { and }\left(p_{1} \cdot \ldots \cdot p_{s}\right)^{\alpha} F \subset S .
$$

If this is the case, then we say that $S$ is finitely primary of rank $s$ and exponent $\alpha$.

If $s=1$ and $F^{\times}=\{1\}$, then $S$ is isomorphic to a numerical monoid. Furthermore, $S$ is tame if and only if it is of rank 1 ([25, Theorem 3.1.5]).

Example 4.7. Let $S=(\mathbb{N} \times \mathbb{N} \cup\{(0,0)\},+)$. Then $\mathrm{c}(S)=\mathrm{c}_{\mathrm{mon}}(S)=3$ and hence $\Delta(S)=\{1\}$. However, we have $\rho(S)=\infty$ and hence $\omega(S)=\mathrm{t}(S)=\infty$ (for the invariants not defined so far, see the discussion at Inequality 5.1).

Proof. By definition, $S$ is a finitely primary monoid of rank 2 and exponent 1 . It is a special case of the monoid studied in [25. Example 3.1.8], where all assertions have been verified apart from the formula for $\mathrm{c}_{\text {mon }}(S)$. Indeed, it is straightforward that $\mathcal{A}(S)=\{(1, m),(m, 1) \mid m \in \mathbb{N}\}$. Furthermore, every element $a=\left(a_{1}, a_{2}\right) \in S \backslash(\mathcal{A}(S) \cup\{(0,0)\})$ can be written as a sum of two atoms, namely $\left(a_{1}, a_{2}\right)=\left(1, a_{2}-1\right)+\left(a_{1}-1,1\right)$. These two observations easily imply the assertions on $\mathrm{c}(S), \Delta(S)$ and $\rho(S)$. In order to show that $\mathrm{c}_{\text {mon }}(S)=3$ we proceed in two steps.

First we show that $\mathrm{c}_{\text {adj }}(S)=3$. Let $a \in S$ and $k, l \in \mathrm{L}(a)$ be adjacent lengths, say $k<l$. Since $\Delta(S)=\{1\}$, it follows that $l=k+1 \geq 3$. Let $z=u_{1} \cdot \ldots \cdot u_{k+1} \in \mathrm{Z}_{l}(a)$ with $u_{1}, \ldots, u_{k+1} \in \mathcal{A}(S)$. Since $u_{1} u_{2} u_{3}$ is a product of two atoms, say $u_{1} u_{2} u_{3}=v_{1} v_{2}$ with $v_{1}, v_{2} \in \mathcal{A}(S)$, we infer that $z^{\prime}=$ $v_{1} v_{2} u_{4} \cdot \ldots \cdot u_{k+1} \in \mathrm{Z}_{k}(a)$, and hence $3=\mathrm{d}\left(z, z^{\prime}\right)=\mathrm{d}\left(\mathrm{Z}_{k+1}(a), \mathrm{Z}_{k}(a)\right)$. Thus have $\mathrm{c}_{\text {adj }}(a)=3$, and hence we get $\mathrm{c}_{\mathrm{adj}}(S)=3$.

Second, we verify that $\mathrm{c}_{\text {equal }}(S)=3$. Let $a=(m, n) \in S$ and $k \in \mathrm{L}(a)$. We have to show that each two factorizations $z, z^{\prime} \in \mathrm{Z}_{k}(a)$ can be concatenated by a monotone 3-chain of factorizations. By symmetry, we may assume that $m \leq n$, and then we clearly have $k \leq m$. We consider the factorization

$$
z^{*}=(k-2)(\mathbf{1}, \mathbf{1})+(\boldsymbol{m}-\boldsymbol{k}+\mathbf{1}, \mathbf{1})+(\mathbf{1}, \boldsymbol{n}-\boldsymbol{k}+\mathbf{1}) \in \mathrm{Z}_{k}(a) .
$$

Clearly, it is sufficient to show that from every factorization $z \in Z_{k}(a)$ there is a monotone 3-chain of factorizations to $z^{*}$. Let $z \in \mathrm{Z}_{k}(a)$ be given. We proceed by induction on $\mathrm{v}_{(\mathbf{1}, \mathbf{1})}(z)$. If $\mathrm{v}_{(\mathbf{1}, \mathbf{1})}(z)=k-2$, then $z=z^{*}$ and we are done. Suppose that $\mathrm{v}_{(\mathbf{1}, \mathbf{1})}(z)<k-2$. Then there are two atoms $u_{1}=\left(\mathbf{1}, \boldsymbol{a}_{\mathbf{1}}\right)$ and $u_{2}=\left(\mathbf{1}, \boldsymbol{a}_{\mathbf{2}}\right)$ with $a_{1}, a_{2} \in \mathbb{N}_{\geq 2}$ and $\left(u_{1}+u_{2}\right) \mid z$, or there are two atoms $u_{1}=\left(\boldsymbol{a}_{\mathbf{1}}, \mathbf{1}\right)$ and $u_{2}=\left(\boldsymbol{a}_{\mathbf{2}}, \mathbf{1}\right)$ with $a_{1}, a_{2} \in \mathbb{N}_{\geq 2}$ and $\left(u_{1}+u_{2}\right) \mid z$. By symmetry, we may suppose that the first case holds. Then we define $z^{\prime}=-u_{1}-u_{2}+z+(\mathbf{1}, \mathbf{1})+\left(\mathbf{1}, \boldsymbol{a}_{\mathbf{1}}+\boldsymbol{a}_{\mathbf{2}}-\mathbf{1}\right)$. Clearly, we have $z^{\prime} \in \mathrm{Z}_{k}(a), \mathrm{d}\left(z, z^{\prime}\right)=3$, $\mathrm{v}_{(\mathbf{1}, \mathbf{1})}(z)<\mathrm{v}_{(\mathbf{1}, \mathbf{1})}\left(z^{\prime}\right)$, and hence the assertion follows. 


\section{The tame Degrees}

Definition 5.1. Let $S$ be atomic.

1. For $a \in S$ and $x \in \mathrm{Z}(S)$, let $\mathrm{t}(a, x) \in \mathbb{N}_{0} \cup\{\infty\}$ denote the smallest $N \in \mathbb{N}_{0} \cup\{\infty\}$ with the following property :

If $\mathbf{Z}(a) \cap x \mathbf{Z}(S) \neq \emptyset$ and $z \in \mathbf{Z}(a)$, then there exists $z^{\prime} \in \mathbf{Z}(a) \cap x \mathbf{Z}(S)$ such that $\mathrm{d}\left(z, z^{\prime}\right) \leq N$.

2. For subsets $S^{\prime} \subset S$ and $X \subset \mathrm{Z}(S)$, we define

$$
\mathrm{t}\left(S^{\prime}, X\right)=\sup \left\{\mathrm{t}(a, x) \mid a \in S^{\prime}, x \in X\right\} \in \mathbb{N}_{0} \cup\{\infty\},
$$

and for $a \in S$, we set $\mathrm{t}(a, X)=\mathrm{t}(\{a\}, X)$.

3. $S$ is said to be locally tame if $\mathrm{t}(S, u)<\infty$ for all $u \in \mathcal{A}\left(S_{\text {red }}\right)$. We call

$$
\mathrm{t}(S)=\mathrm{t}\left(S, \mathcal{A}\left(S_{\text {red }}\right)\right)=\sup \left\{\mathrm{t}(S, u) \mid u \in \mathcal{A}\left(S_{\text {red }}\right)\right\} \in \mathbb{N}_{0} \cup\{\infty\}
$$

the tame degree of $S$, and $S$ is called tame if $\mathrm{t}(S)<\infty$.

4. We set $\mathrm{a}(S)=\sup \left\{|x| \mid(x, y) \in \mathcal{A}\left(\sim_{S}\right)\right.$ for some $\left.y \in \mathrm{Z}(S)\right\} \in \mathbb{N}_{0} \cup\{\infty\}$.

Let $S$ be reduced and atomic. Local tameness is a central finiteness property in factorization theory, but the finiteness of the tame degree is a rare property (a non-principal order $\mathfrak{o}$ in an algebraic number field is locally tame with finite catenary degree, and it is tame if and only if for every prime ideal $\mathfrak{p}$ containing the conductor there is precisely one prime ideal $\overline{\mathfrak{p}}$ in the principal order $\overline{\mathfrak{o}}$ such that $\overline{\mathfrak{p}} \cap \mathfrak{o}=\mathfrak{p})$. Whereas in $v$-noetherian monoids (these are monoids satisfying the ascending chain condition for $v$-ideals) we have $\omega(S, u)<\infty$ for all atoms $u \in \mathcal{A}(S)$, this does not hold for the $\mathrm{t}(S, u)$ values (see [28, Corollary 3.6], [27, Theorems 4.2 and 4.4], [26, Theorems 5.3 and 6.7]). However, we have $\omega(S)<\infty$ if and only if $\mathrm{t}(S)<\infty$ (see Inequality 5.1 below). A main aim in this section is to show that monoids having a generic presentation satisfy $\omega(S)=\mathrm{t}(S)$ (Theorem 5.6 and its corollaries). After that we provide the first examples of numerical monoids $S$ with $\omega(S)<\mathrm{t}(S)$.

Let $S$ be atomic and, to avoid trivialities, suppose that $S$ is not factorial. Let us consider a $(S)$. If $S$ is finitely generated, then $\sim_{S}$ is finitely generated, hence $\mathcal{A}\left(\sim_{S}\right)$ is finite, and thus a $(S)<\infty$. It has been proved that $\mathrm{a}(S)$ is an upper bound for a variety of arithmetical invariants, such as the catenary degree (e.g., [35, Proposition 14]; see also the forthcoming Corollary 6.4). However, we have

$$
2+\sup \Delta(S) \stackrel{(1)}{\leq} \mathrm{c}(S) \stackrel{(2)}{\leq} \omega(S) \stackrel{(3)}{\leq} \mathrm{t}(S) \stackrel{(4)}{\leq} \omega(S)^{2} \quad \text { and } \quad \rho(S) \stackrel{(5)}{\leq} \omega(S)
$$

where $\rho(S)$ is the elasticity (see Definition 6.1): for (1) see [25, Theorem 1.6.3], for (2), (4) and (5) see [29, Section 3], and (3) can be found in [27, Theorem 3.6]. In the next proposition we will verify that a $(S)$ is an upper bound for the tame degree $\mathrm{t}(S)$, but even this inequality can be strict (even for numerical monoids, see [8, Example 4.4]).

Proposition 5.2. Let $S$ be atomic.

1. For every $u \in \mathcal{A}\left(S_{\text {red }}\right)$ we have

$$
\mathrm{t}(S, u) \leq \sup \left\{|x|,|y| \mid(x, y) \in \mathcal{A}\left(\sim_{S}\right), x \in u Z(S)\right\} .
$$

2. $\mathrm{t}(S) \leq \mathrm{a}(S)$.

3. If $\mathrm{a}(S)<\infty$, then $\mathrm{c}_{\mathrm{adj}}(S)<\infty$.

Proof. 1. and 2. Obviously, it is sufficient to prove the first statement. Furthermore, we may assume that $S$ is reduced.

Let $u \in \mathcal{A}(S), a \in u S$ and $z \in \mathbf{Z}(a)$. We have to find a factorization $z^{\prime} \in \mathbf{Z}(a) \cap u Z(S)$ such that

$$
\mathrm{d}\left(z, z^{\prime}\right) \leq \sup \left\{|x|,|y| \mid(x, y) \in \mathcal{A}\left(\sim_{S}\right), x \in u Z(S)\right\} \text {. }
$$


Since $a \in u S$, there exists some $\bar{z} \in \mathbf{Z}(a) \cap u \mathrm{Z}(S)$. We consider a factorization of $(z, \bar{z})$ in $\sim_{S}$, say

$$
(z, \bar{z})=\left(z_{1}, \overline{z_{1}}\right) \cdot \ldots \cdot\left(z_{k}, \overline{z_{k}}\right)
$$

where $k \in \mathbb{N},\left(z_{i}, \overline{z_{i}}\right) \in \mathcal{A}\left(\sim_{S}\right)$ for all $i \in[1, k]$ and $\overline{z_{1}} \in u \mathrm{Z}(S)$. Then $z^{\prime}=\overline{z_{1}}\left(z_{1}^{-1} z\right) \in \mathrm{Z}(a) \cap u \mathrm{Z}(S)$ and

$$
\mathrm{d}\left(z, z^{\prime}\right) \leq \max \left\{\left|z_{1}\right|,\left|\overline{z_{1}}\right|\right\} \leq \sup \left\{|x|,|y| \mid(x, y) \in \mathcal{A}\left(\sim_{S}\right), x \in u Z(S)\right\} .
$$

3. Suppose that $\mathrm{a}(S)<\infty$. Then

$$
B=\left\{|x|-|y| \mid(x, y) \in \mathcal{A}\left(\sim_{S}\right)\right\} \subset \mathbb{Z}
$$

is finite. Furthermore, by 2. and Inequality [5.1, the set of distances $\Delta(S)$ is finite. By Proposition 4.4 4, it suffices to verify that

$$
\sup \left\{|x| \mid x \in \operatorname{Min}\left(A_{d}\right), d \in \Delta(S)\right\}<\infty,
$$

where $A_{d}=\{x \in \mathbf{Z}(S)|d+| x \mid \in \mathrm{L}(\pi(x))\}$. Let $x \in \mathbf{Z}(S)$ and $y \in \mathbf{Z}(\pi(x))$ such that $|y|=|x|+d$. Consider a factorization

$$
(x, y)=\left(x_{1}, y_{1}\right) \cdot \ldots \cdot\left(x_{k}, y_{k}\right)
$$

where $k \in \mathbb{N}$ and $\left(x_{i}, y_{i}\right) \in \mathcal{A}\left(\sim_{S}\right)$ for all $i \in[1, k]$. There exists a bound $M(B, d)$ with the following property (for the construction of an explicit upper bound, see [22, Lemma 5.1]): there is a subset $I \subset[1, k]$ with $|I| \leq M(B, d)$ such that for

$$
x^{\prime}=\prod_{i \in I} x_{i} \quad \text { and } \quad y^{\prime}=\prod_{i \in I} y_{i}
$$

we have $\left|y^{\prime}\right|=\left|x^{\prime}\right|+d$. Since $x^{\prime} \mid x$, there is an $x^{*} \in \operatorname{Min}\left(A_{d}\right)$ with $x^{*}\left|x^{\prime}\right| x$ and $\left|x^{*}\right| \leq\left|x^{\prime}\right| \leq M(B, d) \mathrm{a}(S)$.

We discuss the relationship between the finiteness of the monotone catenary degree, of the tame degree and of its upper bound a $(S)$. Example 4.7 shows that the monotone catenary degree can be finite even if the monoid is not tame. Conversely, Example 4.5 in [14 provides a finitely primary monoid of rank 1 (hence it is tame and has finite catenary degree) for which cequal $(S)$ is infinite. In contrast to that example, Theorem 5.1 from [29] shows that a slightly weaker variant of $\mathrm{c}_{\text {adj }}(S)$ is finite for tame monoids. More precisely, it states that in a tame monoid $S$ there is a constant $M \in \mathbb{N}$ with the following property:

For each two adjacent lengths $k, l \in \mathrm{L}(a) \cap[\min \mathrm{L}(a)+M, \max \mathrm{L}(a)-M]$ we have $\mathrm{d}\left(\mathrm{Z}_{k}(a), \mathrm{Z}_{l}(a)\right) \leq M$.

Proposition 5.2. 3 shows that the finiteness of a $(S)$-which is stronger than the finiteness of the tame degree - enforces the finiteness of $\mathrm{c}_{\mathrm{adj}}(S)$. It is an interesting open problem whether the finiteness of the tame degree is strong enough to guarantee the finiteness of $\mathrm{c}_{\text {adj }}(S)$.

Let $S$ be atomic but not factorial. We discuss the relationship between $\omega(S)$ and $\mathrm{t}(S)$. If $u \in S$ is an atom but not prime, then

and thus

$$
\mathrm{t}\left(S, u S^{\times}\right)=\max \{\omega(S, u), 1+\tau(S, u)\} \in \mathbb{N}_{\geq 2} \cup\{\infty\}
$$

$$
\mathrm{t}(S)=\max \{\omega(S), 1+\tau(S)\}
$$

(see [27, Theorem 3.6]; since it is not needed in the sequel, we do not repeat the definition of the $\tau$ invariant). For a large class of Krull monoids it was found out that $\mathrm{t}(S)=1+\tau(S)$ ([27, Corollary 4.6]). In contrast to that result, M. Omidali recently proved that $\omega(S)=\mathrm{t}(S)$ for numerical monoids generated by almost arithmetical progressions (see [32, Theorem 3.10]). Theorem 5.6 provides a result of this type in a more general setting. We will frequently make use of the following fact: if $\mathrm{t}(S)<\infty$, then there is an $a \in S$ and a $u \in \mathcal{A}\left(S_{\text {red }}\right)$ such that $\mathrm{t}(a, u)=\mathrm{t}\left(a, \mathcal{A}\left(S_{\text {red }}\right)\right)=\mathrm{t}(S)$.

Lemma 5.3. Let $S$ be a reduced and finitely generated. Then for every subset $X \subset S$ there exists a finite set $E \subset X$ such that $X \subset E H$. Clearly, $E$ can be chosen to be minimal. 
Proof. This is a special case of [25, Proposition 2.7.4].

The next lemma is a generalization of Lemma 5 in 8$]$.

Lemma 5.4. Let $S$ be reduced and atomic, $a \in S, z \in \mathrm{Z}(a)$ and $u, v \in \mathcal{A}(S)$.

1. Suppose that $\mathrm{t}(a, \mathcal{A}(S))=\mathrm{d}(z, \mathrm{Z}(a) \cap u \mathrm{Z}(S))>0, z \in v \mathrm{Z}(S)$ and $a \in$ uvS. Then $\mathrm{t}\left(v^{-1} a, \mathcal{A}(S)\right) \geq$ $\mathrm{t}(a, \mathcal{A}(S))$.

2. Let $a \in S$ be minimal such that $\mathrm{t}(a, \mathcal{A}(S))=\mathrm{t}(S)>0$ (this means that no proper divisor $b$ of a satisfies $\mathrm{t}(b, \mathcal{A}(S))=\mathrm{t}(S))$ and let $z^{\prime} \in \mathrm{Z}(a) \cap u \mathrm{Z}(S)$ such that $\mathrm{d}\left(z, z^{\prime}\right)=\mathrm{t}(S)$. Then $z \in \operatorname{Min}(\mathrm{Z}(u S))$.

Proof. 1. By definition, we have $\mathrm{t}\left(v^{-1} a, \mathcal{A}(S)\right) \geq \mathrm{d}\left(v^{-1} z, \mathrm{Z}\left(v^{-1} a\right) \cap u \mathrm{Z}(S)\right)$. If $z^{\prime} \in \mathrm{Z}\left(v^{-1} a\right) \cap u \mathrm{Z}(S)$ such that $\mathrm{d}\left(v^{-1} z, z^{\prime}\right)=\mathrm{d}\left(v^{-1} z, \mathrm{Z}\left(v^{-1} a\right) \cap u \mathrm{Z}(S)\right)$, then

$$
\begin{aligned}
\mathrm{t}\left(v^{-1} a, \mathcal{A}(S)\right) & \geq \mathrm{d}\left(v^{-1} z, z^{\prime}\right)=\mathrm{d}\left(z, v z^{\prime}\right)=\mathrm{d}\left(z, v\left(\mathrm{Z}\left(v^{-1} a\right) \cap u \mathrm{Z}(S)\right)\right) \\
& \geq \mathrm{d}(z, \mathrm{Z}(a) \cap u \mathrm{Z}(S))=\mathrm{t}(a, \mathcal{A}(S)) .
\end{aligned}
$$

2. Assume to the contrary that $z \notin \operatorname{Min}(\mathrm{Z}(u S))$. Then there exists an atom, say $v$, such that $v^{-1} z \in$ $\mathrm{Z}(u S)$. Since $z^{\prime} \in u \mathrm{Z}(S)$ with $\mathrm{d}\left(z, z^{\prime}\right)=\mathrm{t}(S)>0$, it follows that $u \nmid z$ and hence $\mathrm{d}(z, \mathrm{Z}(a) \cap u \mathrm{Z}(S))>0$. Thus 1. implies that $\mathrm{t}\left(v^{-1} a, \mathcal{A}(S)\right) \geq \mathrm{t}(a, \mathcal{A}(S))=\mathrm{t}(S)$, a contradiction to the minimality of $a$.

We say that $S$ has a unique minimal presentation if it has a minimal presentation $\sigma$ and for each minimal presentation $\tau$ we have $\sigma \cup \sigma^{-1}=\tau \cup \tau^{-1}$. If this holds then $\sigma$ is called a unique minimal presentation of $S$. We will need that every generic presentation is a unique minimal presentation. Although this can be obtained as a consequence of a result by I.Peeva and B.Sturmfels, we present a short and independent proof in the language of monoids (see [33, Remark 4.4.3]; indeed the term generic presentation has been chosen to reflect the origins in generic lattice ideals).

Proposition 5.5. Every generic presentation of $S$ is a unique minimal presentation.

Proof. We may suppose that $S$ is reduced. Then $S$ is finitely generated. Recall that any minimal presentation is constructed by choosing pairs of elements in different $\mathcal{R}$-classes of elements with more than one $\mathcal{R}$-class (see [36, Chapter 9]).

Let $\sigma \subset \sim_{S}$ be a generic presentation. Then every pair $(x, y) \in \sigma$ has full support, and $x$ and $y$ are in different $\mathcal{R}$-classes. Thus, if $x, y \in \mathbf{Z}(s)$, then $\mathrm{Z}(s)$ can consist of only two $\mathcal{R}$-classes, and the union of their support is the set of all atoms. So for every $s \in S$ with $|\mathrm{Z}(s)| \geq 2$, the set of factorizations $\mathrm{Z}(s)$ consists of precisely two $\mathcal{R}$-classes, and $\sigma$ is unique if and only if each such $\mathcal{R}$-class contains precisely one factorization.

Assume to the contrary that there is an $s \in S$ such that $\mathrm{Z}(s)$ consists of two $\mathcal{R}$-classes and that two distinct factorizations $z, z^{\prime} \in \mathbf{Z}(s)$ are in the same $\mathcal{R}$-class. By definition, $z$ and $z^{\prime}$ can be concatenated by a chain of factorizations $z=z_{0}, \ldots, z_{k}=z^{\prime} \operatorname{such}$ that $\pi\left(z_{i}\right)=s$ and $\operatorname{gcd}\left(z_{i-1}, z_{i}\right) \neq 1$ for all $i \in[1, k]$.

We set $z_{1}=x y_{1}, z_{2}=x y_{2}$ and $s_{1}=\pi\left(y_{1}\right) \in S$ where $x=\operatorname{gcd}\left(z_{1}, z_{2}\right)$ and $y_{1}, y_{2} \in \mathrm{Z}(S)$. Note that $s_{1}$ is a proper divisor of $s_{0}=s . \operatorname{Since} \operatorname{supp}\left(y_{1}\right) \subset \operatorname{supp}\left(z_{1}\right)$ and $\operatorname{supp}\left(y_{2}\right) \subset \operatorname{supp}\left(z_{2}\right), y_{1}$ and $y_{2}$ are in the same $\mathcal{R}$-class, because otherwise $\mathrm{Z}\left(s_{1}\right)$ would consist of two $\mathcal{R}$-classes and there would be a relation without full support.

Iterating this construction we obtain an infinite sequence $\left(s_{i}\right)_{i \geq 0}$ where $s_{i+1}$ is a proper divisor of $s_{i}$ for all $i \in \mathbb{N}_{0}$, a contradiction to $S$ being finitely generated.

Theorem 5.6. Let $S$ be atomic, $P \subset S$ a set of representatives of the set of primes of $S$ and $T$ the set of all $a \in S$ such that $p \nmid a$ for all $p \in P$. Suppose that $T=\coprod_{i \in I} T_{i}, T \neq T^{\times}$and that there is an $i^{*} \in I$ such that $T_{i^{*}}$ has a generic presentation and $\mathrm{t}\left(T_{i^{*}}\right)=\mathrm{t}(T)$. Then $\mathrm{c}(S)=\omega(S)=\mathrm{t}(S)$. 
Remark. Since $\mathrm{t}(T)=\sup \left\{\mathrm{t}\left(T_{i}\right) \mid i \in I\right\}$ ([25, Proposition 1.6.8]), the assumption is of course satisfied if all $T_{i}$ have generic presentations.

Proof. By [25, Theorem 1.2.3], $T \subset S$ is an atomic submonoid and $S=\mathcal{F}(P) \times T$. Note that neither $S$ nor $T$ are factorial because $T \neq T^{\times}$. Since

$$
\mathrm{c}\left(T_{i^{*}}\right) \leq \mathrm{c}(T)=\mathrm{c}(S) \leq \omega(S) \leq \mathrm{t}(S)=\sup \left\{\mathrm{t}\left(T_{i}\right) \mid i \in I\right\}=\mathrm{t}\left(T_{i^{*}}\right),
$$

it suffices to show that $\mathrm{c}\left(T_{i^{*}}\right)=\omega\left(T_{i^{*}}\right)=\mathrm{t}\left(T_{i^{*}}\right)$. Thus, after a change of notation, we may assume that $S$ is reduced, not factorial and has a generic presentation. Let $\sigma \subset \sim_{S}$ denote this generic presentation of $S$. We start with the following assertion.

A. For every $u \in \mathcal{A}(S)$ we have

$$
\operatorname{Min}(\mathrm{Z}(u S))=\{u\} \cup\left\{x \in \mathrm{Z}(S) \mid(x, y) \in \sigma \cup \sigma^{-1} \text { for some } y \in \mathrm{Z}(u S)\right\} .
$$

Proof of A. Since $u \in \operatorname{Min}(\mathrm{Z}(u S))$, we may focus on the elements different from $u$. Let $x=\prod_{v \in \mathcal{A}(S)} v^{k_{v}} \in$ $\operatorname{Min}(\mathrm{Z}(u S)) \backslash\{u\}$ with $k_{v} \in \mathbb{N}_{0}$ for all $v \in \mathcal{A}(S)$, and set $a=\pi(x) \in S$. Since $x \neq u$, it follows that $k_{u}=0$. There exists a $y \in \mathbf{Z}(a) \cap u \mathrm{Z}(S)$, and the pair $(x, y)$ belongs to the congruence generated by $\sigma$. Hence there exists $\left(x^{\prime}, y^{\prime}\right) \in \sigma \cup \sigma^{-1}$ with $x^{\prime} \mid x$. Since $u \nmid x$, we get that $u \nmid x^{\prime}$ and because $\sigma$ is generic, it follows that $u \mid y^{\prime}$ and hence $y^{\prime} \in \mathrm{Z}(u S)$. Since $x \in \operatorname{Min}(\mathrm{Z}(u S)) \backslash\{u\}$, we infer that $x=x^{\prime}$. The uniqueness property of a generic presentation implies that $\mathrm{Z}(a)=\left\{x^{\prime}, y^{\prime}\right\}$. Thus we get that $y=y^{\prime}$ and hence $(x, y)=\left(x^{\prime}, y^{\prime}\right) \in \sigma$.

Conversely, let $x \in \mathbf{Z}(S)$ and $y \in \mathbf{Z}(u S)$ such that $(x, y) \in \sigma \cup \sigma^{-1}$. Then we clearly have $x \in \mathbf{Z}(u S)$, and assume to the contrary that it is not minimal. Then there is an $x^{\prime}=\prod_{v \in \mathcal{A}(S)} v^{k_{v}^{\prime}} \in \mathrm{Z}(u S)$, where $k_{v}^{\prime} \in \mathbb{N}_{0}$ for all $v \in \mathcal{A}(S)$, and with $x^{\prime} \mid x$ and $x^{\prime} \neq x$. Note that $k_{u}^{\prime}=0$. Since $\sigma$ is generic, there exists a $y^{\prime}=\prod_{v \in \mathcal{A}(S)} v^{l_{v}}$, where all $l_{v} \in \mathbb{N}_{0}$, with $l_{u} \neq 0$ and $\pi\left(x^{\prime}\right)=\pi\left(y^{\prime}\right)$. Since the pair $\left(x^{\prime}, y^{\prime}\right)$ is in the congruence generated by $\sigma$, there exist $\left(x^{\prime \prime}, y^{\prime \prime}\right) \in \sigma \cup \sigma^{-1}$ such that $x^{\prime \prime} \mid x^{\prime}$. This implies that $x^{\prime \prime} \mid x$ and $x^{\prime \prime} \neq x$. This contradicts the fact, that elements whose factorizations appear in a generic presentation are not comparable ([20, Corollary 6$])$.

Since $\sigma$ is a generic presentation, for every $u \in \mathcal{A}(S)$ and every $(x, y) \in \sigma$ we have $u \mid x$ or $u \mid y$. Thus A and Proposition 3.3 imply that

$$
\omega(S)=\max \{\max \{|x|,|y|\} \mid(x, y) \in \sigma\} .
$$

Now the minimality property of a generic presentation (see Proposition [5.5]), the above formula for $\omega(S)$ together with Proposition 4.6 imply that $\omega(S)=\mathrm{c}(S)$.

Since $\omega(S) \leq \mathrm{t}(S)$, it remains to show that converse inequality. Let $a \in S$ be minimal such that $\mathrm{t}(a, \mathcal{A}(S))=\mathrm{t}(S)$, and let $u \in \mathcal{A}(S), z \in \mathrm{Z}(a)$ and $z^{\prime} \in \mathrm{Z}(a) \cap u \mathrm{Z}(S)$ such that

$$
\mathrm{t}(S)=\mathrm{t}(a, \mathcal{A}(S))=\mathrm{d}\left(z, z^{\prime}\right)
$$

By Lemma 5.4, it follows that $z \in \operatorname{Min}(\mathbf{Z}(u S))$. Thus, by A, there exist $(x, y) \in \sigma \cup \sigma^{-1}$ such that $y=z$. Therefore $x$ and $y$ are factorizations of $a$, which appear in the unique presentation of $S$. This implies that $\mathrm{Z}(a)=\{x, y\}$, and thus $z^{\prime}=x$ and $\mathrm{t}(S)=\mathrm{d}\left(z, z^{\prime}\right) \leq \max \{|x|,|y|\} \leq \omega(S)$.

Let $R$ be an integral domain. We denote by $R^{\bullet}=R \backslash\{0\}$ its multiplicative monoid of non-zero elements, by $\mathfrak{X}(R)$ the set of all minimal non-zero prime ideals of $R$, by $\widehat{R}$ its complete integral closure, and by $(R: \widehat{R})=\{f \in R \mid f \widehat{R} \subset R\}$ the conductor of $R$ in $\widehat{R}$.

Corollary 5.7. Let $R$ be a weakly Krull domain, $\mathfrak{f}=(R: \widehat{R}) \neq\{0\}, \mathcal{P}^{*}=\{\mathfrak{p} \in \mathfrak{X}(R) \mid \mathfrak{p} \supset \mathfrak{f}\}$ and $S=\mathcal{I}_{v}^{*}(R)$ the monoid of $v$-invertible $v$-ideals equipped with $v$-multiplication. If for every $\mathfrak{p} \in \mathcal{P}^{*}$, the monoid $R_{\mathfrak{p}} \bullet$ has a generic presentation, then $\mathrm{c}(S)=\omega(S)=\mathrm{t}(S)$. 
Proof. By [25, Theorem 3.7.1], the monoid $S$ is isomorphic to $\mathcal{F}(P) \times T$, where

$$
\mathcal{P}=\{\mathfrak{p} \in \mathfrak{X}(R) \mid \mathfrak{p} \not \supset \mathfrak{f}\} \quad \text { and } \quad T=\prod_{\mathfrak{p} \in \mathcal{P}^{*}}\left(R_{\mathfrak{p}}^{\bullet}\right)_{\text {red }}
$$

Thus the assertion follows from Theorem 5.6.

Let all notations be as in Corollary 5.7. It is easy to point out explicit examples where the assumptions hold (for details see [25, Section 3.7]). Every one-dimensional noetherian domain, in particular every order in a Dedekind domain, is weakly Krull. Let $\mathfrak{p} \in \mathcal{P}^{*}$. Then $R_{\mathfrak{p}} \bullet$ is finitely primary, and $R_{\mathfrak{p}} \bullet$ is tame if and only if there exists precisely one prime ideal $\widehat{\mathfrak{p}} \in \mathfrak{X}(\widehat{R})$ satisfying $\widehat{\mathfrak{p}} \cap R=\mathfrak{p}$. Suppose this holds true, and set $H=\left(R_{\mathfrak{p}}^{\bullet}\right)_{\text {red. }}$ Then $H \subset F=F^{\times} \times[p]$ where $p$ is a prime element of the factorial monoid $F$, and its value monoid $\mathrm{v}_{p}(H)=\left\{\mathrm{v}_{p}(a) \mid a \in H\right\} \subset\left(\mathbb{N}_{0},+\right)$ is a numerical monoid. If $R$ is a non-principal order in an algebraic number field, then $F^{\times}$is finite.

Corollary 5.8. Let $S$ be a numerical monoid with $\mathcal{A}(S)=\left\{n_{1}, n_{2}, n_{3}\right\}$ where $\operatorname{gcd}\left(n_{1}, n_{2}\right)=\operatorname{gcd}\left(n_{1}, n_{3}\right)=$ $\operatorname{gcd}\left(n_{2}, n_{3}\right)=1$. Then $S$ has a generic presentation and $\mathrm{c}(S)=\omega(S)=\mathrm{t}(S)$. More precisely, if

$$
c_{i}=\min \left\{k \in \mathbb{N} \mid k n_{i} \in\left\langle n_{j}, n_{k}\right\rangle\right\} \quad \text { and } \quad c_{i} n_{i}=r_{i, j} n_{j}+r_{i, k} n_{k},
$$

where $\{i, j, k\}=\{1,2,3\}$ and $r_{i, j} \in \mathbb{N}_{0}$, then $\mathrm{c}(S)=\max \left\{c_{1}, c_{2}, c_{3}, r_{12}+r_{1,3}, r_{2,1}+r_{2,3}, r_{3,1}+r_{3,2}\right\}$.

Proof. $S$ has a generic presentation by [37, Lemma 10.18] and hence Theorem 5.6 implies that $\mathrm{c}(S)=$ $\omega(S)=\mathrm{t}(S)$. The formula for $\mathrm{c}(S)$ stems from [37, Exercise 8.23] (note that the integers $r_{i, j}$ are uniquely determined).

The catenary degree of numerical monoids with embedding dimension three has been also described (with a different approach) in [1]. Let $S$ be atomic. Obviously, the requirement that $S$ has a generic presentation (enforcing $\mathrm{c}(S)=\mathrm{t}(S)$ ) is a strong assumption, and also the general philosophy in factorization theory confirms the idea that the equality of the catenary and the tame degree should be an exceptional phenomenon (see also Corollary (5.10). On the other hand, all types of numerical monoids studied so far share this exceptional phenomenon. This contrast will become more clear in the following remark, where we also construct the first infinite family of numerical monoids whose catenary degrees are strictly smaller than the tame degrees.

\section{Remarks 5.9.}

1. Let $S$ be a numerical monoid with $\mathcal{A}(S)=\left\{n_{1}, \ldots, n_{t}\right\}$, where $t \in \mathbb{N}$ and $1<n_{1}<\ldots<n_{t}$, and let $s \in S$. Consider a factorization $z=a_{1} \mathbf{n}_{1}+\cdots+a_{t} \mathbf{n}_{t} \in \operatorname{Min}(\mathrm{Z}(s+S))$. Then $z \in \mathrm{Z}(a)$ where $a=a_{1} n_{1}+\ldots+a_{t} n_{t}$, and $a=s+u$ for some $u \in S$. Pick some $j \in[1, t]$ such that $\mathbf{n}_{j} \mid z$. The minimality of $z$ implies that $z-\mathbf{n}_{j} \notin \mathrm{Z}(s+S)$, and thus $a-s-n_{j}=u-n_{j} \notin S$ whence $u \in \operatorname{Ap}\left(S, n_{j}\right)$. Thus, if we want to compute the elements in $\operatorname{Min}(\mathrm{Z}(s+S)$ ) (which in view of Proposition 3.3 enables us to determine $\omega(S)$ ), we only have to find the factorizations of the elements of the form $s+u$ with $u$ in the Apéry set of some atom.

We implemented this procedure in GAP by using the numericalsgps package (see [13). We did an exhaustive search computing all numerical monoids with Frobenius number up to 20. That makes 3515 numerical monoids, and the only monoids $S$ in this set fulfilling $\omega(S)<\mathrm{t}(S)$ are $\langle 5,6,9\rangle,\langle 5,8,12\rangle$ and $\langle 6,8,9\rangle$.

2. The minimal presentations of the above three numerical monoids are very similar. Playing around with the Smith normal form of the matrix whose rows are the differences of the relators of these monoids, one can find even wilder examples. The monoid $S=\langle 19,46,391\rangle$ has $\omega(S)=23<39=\mathrm{t}(S)$.

3. We present an infinite family of numerical monoids whose $\omega$-invariants are strictly smaller than the tame degrees. Let $q$ be a prime, $p_{1}, p_{2} \in \mathbb{N}$ with $p_{1}<p_{2}, p_{1}+p_{2}=q$ and $\operatorname{gcd}\left(p_{1}, p_{2}\right)=1$, and 
$k \in \mathbb{N}_{\geq 2} \backslash q \mathbb{N}$ such that $p_{1} k<q<p_{2} k$. We define

$$
S_{k}=\left\langle p_{1} k, q, p_{2} k\right\rangle,
$$

and set $n_{1}=p_{1} k, n_{2}=q, n_{3}=p_{2} k$ and $c_{i}=\min \left\{m \in \mathbb{N} \mid m n_{i} \in\left\langle n_{j}, n_{k}\right\rangle\right\}$ with $\{i, j, k\}=\{1,2,3\}$ (note that $c_{1}, c_{2}$ and $c_{3}$ are as in Corollary 5.8 ).

(a) The Diophantine equation $q x+p_{2} k y=p_{2} k t$ has general solution $x=k t-p_{2} k s, y=-t+q s$, $s \in \mathbb{Z}$. The first $t$ for which $x$ and $y$ can be non-negative is $t=p_{2}$. This in particular means that $p_{1} k$ is not in $\left\langle q, p_{2} k\right\rangle$ and that $c_{1}=p_{2}$. In fact, $p_{2} n_{1}=p_{1} n_{3}$, and $\left(p_{2}+1\right) n_{1}=k n_{2}+\left(p_{1}-1\right) n_{3}$.

(b) Analogously one proves that $c_{2}=k ; k n_{2}=n_{1}+n_{3}$.

(c) It is also easy to show that $c_{3}=p_{1}: p_{1} n_{3}=p_{2} n_{1}$. Moreover, $\left(p_{1}+1\right) n_{3}=\left(p_{2}-1\right) n_{1}+k n_{2}$. By using this information it easily follows that

$$
\begin{gathered}
\operatorname{Min}\left(\mathbf{Z}\left(n_{1}+S_{k}\right)\right)=\left\{\mathbf{n}_{1}, k \mathbf{n}_{2}, p_{1} \mathbf{n}_{3}\right\}, \\
\operatorname{Min}\left(\mathbf{Z}\left(n_{2}+S_{k}\right)\right)=\left\{\left(p_{2}+1\right) \mathbf{n}_{1}, \mathbf{n}_{1}+\mathbf{n}_{3}, \mathbf{n}_{2},\left(p_{1}+1\right) \mathbf{n}_{3}\right\}, \\
\operatorname{Min}\left(\mathbf{Z}\left(n_{3}+S_{k}\right)\right)=\left\{p_{2} \mathbf{n}_{1}, k \mathbf{n}_{2}, \mathbf{n}_{3}\right\} .
\end{gathered}
$$

Therefore Proposition 3.3 implies that

$$
\omega\left(S_{k}\right)=\max \left\{k, p_{2}+1\right\} .
$$

Note that $\mathbf{Z}\left(\left(p_{1}+1\right) n_{3}\right)=\left\{\left(p_{1}+1\right) \mathbf{n}_{3},\left(p_{2}-1\right) \mathbf{n}_{1}+k \mathbf{n}_{2}, p_{2} \mathbf{n}_{1}+\mathbf{n}_{3}\right\}$. Thus, analyzing the factorizations of the elements in $\pi\left(\operatorname{Min}\left(\mathrm{Z}\left(n_{i}+S_{k}\right)\right)\right)$ for $i \in[1,3]$, and by using 1 ., we obtain that

$$
\mathrm{t}\left(S_{k}\right)=\max \left\{\mathrm{t}\left(\left(p_{2}+1\right) e_{1}, \mathcal{A}\left(S_{k}\right)\right), \mathrm{t}\left(\left(p_{1}+1\right) e_{3}, \mathcal{A}\left(S_{k}\right)\right)\right\}=\max \left\{p_{2}+1, k+p_{2}-1\right\}=k+p_{2}-1,
$$

which is strictly larger than $\omega\left(S_{k}\right)$. Furthermore, if $k \geq p_{2}+1$, then

$$
1<\frac{\mathrm{t}\left(S_{k}\right)}{\omega\left(S_{k}\right)}=1+\frac{p_{2}-1}{k} \leq 1+\frac{p_{2}-1}{p_{2}+1}<2 .
$$

We end this section with a brief glance at Krull monoids. For them the equivalence of the catenary and the tame degree is an even rarer phenomenon than it is for numerical monoids. Let $S$ be a Krull monoid with class group $G$ and let $G_{P} \subset G$ denote the set of classes containing prime divisors. If $\mathrm{D}\left(G_{P}\right)<\infty$, then $S$ is tame, and the converse holds - among others - if $S$ the multiplicative monoid of non-zero elements of a domain (see [29, Theorem 4.2]). If $G$ is finite with $|G| \geq 3$ and $G_{P}=G$, then [25, Corollary 3.4.12] shows that

$$
\mathrm{c}(S)=\mathrm{c}(G) \leq \mathrm{D}(G)=\omega(S) \leq \mathrm{t}(G) \leq \mathrm{t}(S),
$$

where the final inequality can be strict ([25, Example 3.4.14]).

Corollary 5.10. Let $G$ be a finite abelian group with $|G| \geq 3$.

1. $\mathrm{c}(G)=\mathrm{t}(G)$ if and only if $G \in\left\{C_{3}, C_{4}, C_{2}^{2}, C_{2}^{3}\right\}$.

2. The monoid of zero-sum sequences $\mathcal{B}\left(G^{\bullet}\right)$ has a generic presentation if and only if $G \in\left\{C_{3}, C_{2}^{2}\right\}$.

Proof. 1. See [25, Corollary 6.5.7].

2. By 1. and by Theorem 5.6, we have to check only the groups in $\left\{C_{3}, C_{4}, C_{2}^{2}, C_{2}^{3}\right\}$. We recall the following facts (for details see [36, Chapter 9]). If $\sigma$ is a minimal presentation for $\mathcal{B}\left(G^{\bullet}\right)$ and $(a, b) \in \sigma$, then $a$ and $b$ are in different $\mathcal{R}$-classes. In fact, any minimal presentation is constructed by choosing pairs of elements in different $\mathcal{R}$-classes of elements with more than one $\mathcal{R}$-class.

If $G=C_{3}=\{0, g, 2 g\}$, then $\mathcal{A}\left(\mathcal{B}\left(G^{\bullet}\right)\right)=\left\{U_{1}=g^{3}, U_{2}=(2 g)^{3}, V=g(2 g)\right\}$ and $\sigma=\left\{\left(U_{1} U_{2}, V^{3}\right)\right\} \subset$ $\sim_{\mathcal{B}(G \bullet)}$ is a generic presentation.

If $G=C_{2} \oplus C_{2}=\left\{0, e_{1}, e_{2}, e_{1}+e_{2}\right\}$, then $\mathcal{A}\left(\mathcal{B}\left(G^{\bullet}\right)\right)=\left\{U_{1}=e_{1}^{2}, U_{2}=e_{2}^{2}, U_{3}=\left(e_{1}+e_{2}\right)^{2}, V=\right.$ $\left.e_{1} e_{2}\left(e_{1}+e_{2}\right)\right\}$ and $\left.\sigma=\left\{\left(U_{1} U_{2} U_{3}, V^{2}\right)\right\} \subset \sim_{\mathcal{B}(G \bullet}\right)$ is a generic presentation. 
Let $G=C_{4}=\{0, g, 2 g,-g\}$. Then $\mathcal{A}\left(\mathcal{B}\left(G^{\bullet}\right)\right)=\left\{U_{1}=g^{4}, U_{2}=(2 g)^{2}, U_{3}=(-g)^{4}, U_{4}=(-g) g, U_{5}=\right.$ $g^{2}(2 g), U_{6}=\left((2 g)(-g)^{2}\right\}$ and $\left.\left(U_{1} U_{3}, U_{4}^{4}\right) \in \sim_{\mathcal{B}(G \bullet}\right)$. Since $\mathbf{Z}\left(U_{4}^{4}\right)=\left\{U_{1} U_{3}, U_{4}^{4}\right\}$, the set of factorizations of $U_{4}^{4}$ has only two $\mathcal{R}$-classes, where each consists of precisely one factorization. Thus $\left(U_{4}^{4}, U_{1} U_{3}\right) \in \sigma \cup \sigma^{-1}$, for every minimal presentation $\sigma$. Obviously, this pair does not have full support, and hence $\mathcal{B}\left(G^{\bullet}\right)$ has no generic presentation.

Let $G=C_{2} \oplus C_{2} \oplus C_{2}=\left\{0, e_{1}, e_{2}, e_{3}, e_{1}+e_{2}, e_{1}+e_{3}, e_{2}+e_{3}, e_{1}+e_{2}+e_{3}\right\}$. Then $U_{1}=e_{1}^{2}, U_{2}=e_{2}^{2}, U_{3}=$ $\left(e_{1}+e_{2}\right)^{2}, V=e_{1} e_{2}\left(e_{1}+e_{2}\right) \in \mathcal{A}\left(\mathcal{B}\left(G^{\bullet}\right)\right)$ and $\left.\left(U_{1} U_{2}, V^{3}\right) \in \sim_{\mathcal{B}(G \bullet}\right)$. Since every minimal presentation $\sigma$ contains the relation $\left(U_{1} U_{2}, V^{3}\right)$ which does not have full support, it follows that $\mathcal{B}\left(G^{\bullet}\right)$ has no generic presentation.

\section{UNIONS OF SETS OF LENGTHS}

Definition 6.1. Let $S$ be atomic and $k \in \mathbb{N}$.

1. If $S=S^{\times}$, we set $\mathcal{V}_{k}(S)=\{k\}$. If $S \neq S^{\times}$, let $\mathcal{V}_{k}(S)$ denote the set of all $m \in \mathbb{N}$ for which there exist $u_{1}, \ldots, u_{k}, v_{1}, \ldots, v_{m} \in \mathcal{A}(S)$ with $u_{1} \cdot \ldots \cdot u_{k}=v_{1} \cdot \ldots \cdot v_{m}$.

2. We define

$$
\rho_{k}(S)=\sup \mathcal{V}_{k}(S) \in \mathbb{N} \cup\{\infty\} \quad \text { and } \quad \lambda_{k}(S)=\min \mathcal{V}_{k}(S) \in[1, k]
$$

3. For $a \in S, \rho(a)=\rho(\mathrm{L}(a))$ is called the elasticity of $a$, and

$$
\rho(S)=\sup \{\rho(L) \mid L \in \mathcal{L}(S)\} \in \mathbb{R}_{\geq 1} \cup\{\infty\}
$$

is called the elasticity of $S$. We say that $S$ has finite accepted elasticity if there exists some $a \in S$ with $\rho(a)=\rho(S)<\infty$.

Let $k, l \in \mathbb{N}$. Then $k \in \mathcal{V}_{k}(S), \mathcal{V}_{k}(S)+\mathcal{V}_{l}(S) \subset \mathcal{V}_{k+l}(S)$,

$$
\lambda_{k+l}(S) \leq \lambda_{k}(S)+\lambda_{l}(S) \leq k+l \leq \rho_{k}(S)+\rho_{l}(S) \leq \rho_{k+l}(S),
$$

and

$$
\rho(S)=\sup \left\{\frac{\rho_{k}(S)}{k} \mid k \in \mathbb{N}\right\}=\lim _{k \rightarrow \infty} \frac{\rho_{k}(S)}{k} \quad \text { and } \quad \frac{1}{\rho(S)}=\inf \left\{\frac{\lambda_{k}(S)}{k} \mid k \in \mathbb{N}\right\}=\lim _{k \rightarrow \infty} \frac{\lambda_{k}(S)}{k},
$$

(see [25, Proposition 1.4.2] and [19, Section 3]). Moreover, if $S \neq S^{\times}$, then

$$
\mathcal{V}_{k}(S)=\bigcup_{k \in L, L \in \mathcal{L}(S)} L
$$

is the union of all sets of lengths containing $k$. These unions were introduced by S.T. Chapman and W.W. Smith in [11]. It was proved only recently that a $v$-noetherian monoid, which satisfies $\rho_{k}(S)<\infty$ for all $k \in \mathbb{N}$, is locally tame (see [27, Corollary 4.3]). For Krull monoids with finite class group, the invariants $\rho_{k}(S)$ are studied in [24].

The first part of this section is devoted to the invariant $\rho_{k}(S)$ in a more general setting, and after that we study the structure of the unions of sets of lengths for numerical monoids.

Proposition 6.2. Let $S$ be atomic with $S \neq S^{\times}$.

1. If $S$ has finite accepted elasticity, then the sets

$$
M=\left\{k \in \mathbb{N} \mid \frac{\rho_{k}(S)}{k}=\rho(S)\right\} \cup\{0\} \quad \text { and } \quad M^{\prime}=\left\{k \in \mathbb{N} \mid \frac{\lambda_{k}(S)}{k}=\frac{1}{\rho(S)}\right\} \cup\{0\}
$$

are submonoids of $\left(\mathbb{N}_{0},+\right)$, distinct from $\{0\}$. 
2. Let $a \in S, z=u_{1} \cdot \ldots \cdot u_{l} \in \mathbf{Z}(a)$ and $z^{\prime}=v_{1} \cdot \ldots \cdot v_{\rho} \in \mathbf{Z}(a)$ where $l \in \mathbb{N}, \rho=\rho_{l}(S)$ and $u_{1} \ldots, u_{l}, v_{1}, \ldots, v_{\rho} \in \mathcal{A}\left(S_{\text {red }}\right)$. If there is no $k \in[1, l-1]$ such that $\rho_{k}(S)+\rho_{l-k}(S)=\rho_{l}(S)$, then $\left(z, z^{\prime}\right) \in \mathcal{A}\left(\sim_{S}\right)$.

Proof. We may suppose that $S$ is reduced.

1. Suppose that $S$ has finite accepted elasticity. First we consider the set $M$. By definition, there is an $a \in S$ such that $\rho(S)=\rho(a)$. If $k=\min \mathrm{L}(a)$ and $\rho=\max \mathrm{L}(a)$, then

$$
\rho(S)=\frac{\rho}{k} \leq \frac{\rho_{k}(S)}{k} \leq \rho(S)
$$

and hence $k \in M$. Let $i \in[1,2]$ and $k_{i} \in M$. Since $\left(k_{1}+k_{2}\right) \rho(S)=\rho_{k_{1}}(S)+\rho_{k_{2}}(S) \leq \rho_{k_{1}+k_{2}}(S)$, it follows that

$$
\rho(S) \geq \frac{\rho_{k_{1}+k_{2}}(S)}{k_{1}+k_{2}} \geq \frac{\rho_{k_{1}}(S)+\rho_{k_{2}}(S)}{k_{1}+k_{2}}=\rho(S) .
$$

Thus equality holds, and $k_{1}+k_{2} \in M$. To verify the assertion on $M^{\prime}$, we choose an $l \in \mathbb{N}$ such that $\rho_{l}(S) / l=\rho(S)$. Then $\lambda_{\rho_{l}(S)}(S) \leq l$, and since

$$
\frac{1}{\rho(S)} \leq \frac{\lambda_{\rho_{l}(S)}(S)}{\rho_{l}(S)} \leq \frac{l}{\rho_{l}(S)}=\frac{1}{\rho(S)},
$$

it follows that $\rho_{l}(S) \in M^{\prime}$. Let $i \in[1,2]$ and $k_{i} \in M^{\prime}$. Since $\left(k_{1}+k_{2}\right) / \rho(S)=\lambda_{k_{1}}(S)+\lambda_{k_{2}}(S) \geq \lambda_{k_{1}+k_{2}}(S)$, it follows that

$$
\frac{1}{\rho(S)} \leq \frac{\lambda_{k_{1}+k_{2}}(S)}{k_{1}+k_{2}} \leq \frac{\lambda_{k_{1}}(S)+\lambda_{k_{2}}(S)}{k_{1}+k_{2}}=\frac{1}{\rho(S)}
$$

Thus equality holds and $k_{1}+k_{2} \in M^{\prime}$.

2. Assume to the contrary that $\left(z, z^{\prime}\right) \notin \mathcal{A}\left(\sim_{S}\right)$. Then there exists $\left(x, x^{\prime}\right) \in \sim_{S}$ such that $\left(x, x^{\prime}\right) \mid\left(z, z^{\prime}\right)$ with $1 \neq\left(x, x^{\prime}\right) \neq\left(z, z^{\prime}\right)$. After renumbering if necessary we may suppose that $x=u_{1} \cdot \ldots \cdot u_{k}$ and $x^{\prime}=v_{1} \cdot \ldots \cdot v_{\psi}$ where $k \in[1, l-1]$ and $\psi \in[1, \rho-1]$. Then $u_{k+1} \cdot \ldots \cdot u_{l}=v_{\psi+1} \cdot \ldots \cdot v_{\rho}$ and

$$
\rho_{l}(S)=\rho=\psi+(\rho-\psi) \leq \rho_{k}(S)+\rho_{l-k}(S) \leq \rho_{l}(S),
$$

a contradiction.

Corollary 6.3. Let $S$ be a numerical monoid with $\mathcal{A}(S)=\left\{n_{1}, \ldots, n_{t}\right\}$ where $t \in \mathbb{N}$ and $1<n_{1}<\ldots<n_{t}$.

1. Then

$$
\rho(S)=\frac{n_{t}}{n_{1}} \quad \text { and } \quad \min \Delta(S)=\operatorname{gcd}\left(n_{2}-n_{1}, \ldots, n_{t}-n_{t-1}\right) .
$$

2. $\left\{k \in \mathbb{N} \mid \frac{\rho_{k}(S)}{k}=\rho(S)\right\} \cup\{0\}=\frac{\operatorname{lcm}\left(n_{1}, n_{t}\right)}{n_{t}} \mathbb{N}_{0}$.

3. $\left\{k \in \mathbb{N} \mid \frac{\lambda_{k}(S)}{k}=\frac{1}{\rho(S)}\right\} \cup\{0\}=\frac{\operatorname{lcm}\left(n_{1}, n_{t}\right)}{n_{1}} \mathbb{N}_{0}$.

Proof. 1. See [10, Theorem 2.1] and [7, Proposition 2.9].

2. Let $a \in \mathbb{N}$ be a multiple of $\operatorname{lcm}\left(n_{1}, n_{t}\right)$. We show that $a / n_{t}$ is in the set on the left hand side. We have

and

$$
a=\frac{a}{n_{1}} \boldsymbol{n}_{\mathbf{1}}=\frac{a}{n_{t}} \boldsymbol{n}_{\boldsymbol{t}}, \quad \min \mathrm{L}(a) \leq \frac{a}{n_{t}}, \quad \max \mathrm{L}(a) \geq \frac{a}{n_{1}}
$$

$$
\frac{n_{t}}{n_{1}}=\rho(S) \geq \rho(a)=\frac{\max \mathrm{L}(a)}{\min \mathrm{L}(a)} \geq \frac{n_{t}}{n_{1}} .
$$

This shows that $\min \mathrm{L}(a)=a / n_{t}, \max \mathrm{L}(a)=a / n_{1}$ and

$$
\frac{n_{t}}{n_{1}}=\rho(a) \leq \frac{\rho_{\min \mathrm{L}(a)}(S)}{\min \mathrm{L}(a)} \leq \rho(S)=\frac{n_{t}}{n_{1}} .
$$

Thus equality holds and $\min \mathrm{L}(a)=a / n_{t}$ has the required property. 
Conversely, let $k \in \mathbb{N}$ with $\rho_{k}(S) / k=\rho(S)=n_{t} / n_{1}$. We choose $a \in \mathcal{V}_{k}(S)$ with $\max \mathrm{L}(a)=\rho_{k}(S)$. Then $\min \mathrm{L}(a) \leq k$ and

$$
\frac{n_{t}}{n_{1}}=\frac{\rho_{k}(S)}{k} \leq \frac{\max \mathrm{L}(a)}{\min \mathrm{L}(a)}=\rho(a) \leq \frac{n_{t}}{n_{1}}
$$

implies that $\min \mathrm{L}(a)=k$. Since $a / n_{t} \leq \min \mathrm{L}(a), \max \mathrm{L}(a) \leq a / n_{1}$ and

$$
\frac{n_{t}}{n_{1}}=\rho(a) \leq \frac{a / n_{1}}{a / n_{t}}=\frac{n_{t}}{n_{1}}
$$

it follows that $n_{1} \mid a$ and $n_{t} \mid a$. Therefore $\operatorname{lcm}\left(n_{1}, n_{t}\right) \mid a$ and

$$
k=\min \mathrm{L}(a)=\frac{a}{n_{t}} \in \frac{\operatorname{lcm}\left(n_{1}, n_{t}\right)}{n_{t}} \mathbb{N}_{0} .
$$

3. Let $a \in \mathbb{N}$ be a multiple of $\operatorname{lcm}\left(n_{1}, n_{t}\right)$. We show that $a / n_{1}$ is in the set on the left hand side which runs along the lines of 2. Conversely, let $k \in \mathbb{N}$ with $\lambda_{k}(S) / k=1 / \rho(S)=n_{1} / n_{t}$. We choose $a \in \mathcal{V}_{k}(S)$ with $\min \mathrm{L}(a)=\lambda_{k}(S)$. Again arguing as in 2., we infer that

$$
k=\max \mathrm{L}(a)=\frac{a}{n_{1}} \in \frac{\operatorname{lcm}\left(n_{1}, n_{t}\right)}{n_{1}} \mathbb{N}_{0} .
$$

Corollary 6.4. Let $S$ be a reduced Krull monoid, $F=\mathcal{F}(P)$ a free monoid such that $S \subset F$ is a saturated and cofinal submonoid, $G=F / S$ and $G_{P}=\{p \mathrm{q}(S) \mid p \in P\} \subset G$ the set of classes containing prime divisors. Suppose that $G_{P}=-G_{P}$ and that $\mathrm{D}\left(G_{P}\right)<\infty$.

1. We have $\rho(S)=\mathrm{D}\left(G_{P}\right) / 2$ and $2 \mathbb{N} \subset\left\{k \in \mathbb{N} \mid \frac{\rho_{k}(S)}{k}=\rho(S)\right\}$.

2. Let $m \in \mathbb{N}$ be minimal such that

$$
\rho_{2 m+1}\left(G_{P}\right)-m \mathrm{D}\left(G_{P}\right)=\max \left\{\rho_{2 k+1}\left(G_{P}\right)-k \mathrm{D}\left(G_{P}\right) \mid k \in \mathbb{N}\right\} .
$$

Then $\rho_{2 m+1}(S) \leq \mathrm{a}\left(\mathcal{B}\left(G_{P}\right)\right)$.

Proof. 1. See [25, Theorem 3.4.10].

2. For every $k \in \mathbb{N}$, we set (as it is usual) $\rho_{k}\left(G_{P}\right)=\rho_{k}\left(\mathcal{B}\left(G_{P}\right)\right.$ ), and by [25, Theorem 3.4.10] we have $\rho_{k}(S)=\rho_{k}\left(G_{P}\right)$. Thus it suffices to verify that $\rho_{2 m+1}\left(G_{P}\right)$ has the asserted upper bound. Let $U_{1}, \ldots, U_{2 m+1}, V_{1}, \ldots, V_{\rho} \in \mathcal{A}\left(\mathcal{B}\left(G_{P}\right)\right)$ with $U_{1} \cdot \ldots \cdot U_{2 m+1}=V_{1} \cdot \ldots \cdot V_{\rho}$ and $\rho=\rho_{2 m+1}\left(G_{P}\right)$. We assert that there is no $k \in[1,2 m]$ such that $\rho_{k}\left(G_{P}\right)+\rho_{2 m+1-k}\left(G_{P}\right)=\rho$. If this holds, then Proposition 6.2 implies that $\left(z=U_{1} \cdot \ldots \cdot U_{2 m+1}, z^{\prime}=V_{1} \cdot \ldots \cdot V_{\rho}\right) \in \mathcal{A}\left(\sim_{\mathcal{B}\left(G_{P}\right)}\right)$ and hence

$$
\begin{aligned}
\rho_{2 m+1}\left(G_{P}\right) & =\rho=\max \{2 m+1, \rho\}=\max \left\{|z|,\left|z^{\prime}\right|\right\} \\
& \leq \sup \left\{|x| \mid(x, y) \in \mathcal{A}\left(\sim_{\mathcal{B}\left(G_{P}\right)}\right) \text { for some } y \in \mathrm{Z}\left(\mathcal{B}\left(G_{P}\right)\right)\right\}=\mathrm{a}\left(\mathcal{B}\left(G_{P}\right)\right) .
\end{aligned}
$$

Assume to the contrary, that there is a $k \in[1,2 m]$ such that $\rho_{k}\left(G_{P}\right)+\rho_{2 m+1-k}\left(G_{P}\right)=\rho_{2 m+1}\left(G_{P}\right)$. Then either $k$ or $2 m+1-k$ are odd, say $k=2 s+1$ with $s \in \mathbb{N}_{0}$. Since, by 1 ., we have $\rho_{2(m-s)}\left(G_{P}\right)=$ $(m-s) \mathrm{D}\left(G_{P}\right)$, we infer that

$$
\rho_{2 s+1}\left(G_{P}\right)-s \mathrm{D}\left(G_{P}\right)=\rho_{2 m+1}\left(G_{P}\right)-\rho_{2(m-s)}\left(G_{P}\right)-s \mathrm{D}\left(G_{P}\right)=\rho_{2 m+1}\left(G_{P}\right)-m \mathrm{D}\left(G_{P}\right),
$$

a contradiction.

Let all notations be as Corollary 6.4 and suppose in addition that $G_{P}=G$ is finite abelian. In all situations studied so far, the set

$$
M=\left\{k \in \mathbb{N} \mid \frac{\rho_{k}(S)}{k}=\rho(S)\right\} \cup\{0\}
$$

contains an odd element, and hence (by Proposition 6.2 and by Corollary $6.4,1$ ) $M$ is a numerical monoid. The standing conjecture is that this holds for all finite abelian groups $G$ (see [24]). 
Next we deal with the structure of the unions of sets of lengths. Suppose $S$ is a Krull monoid such that every class contains a prime divisor. Then it was shown only recently that, for all $k \in \mathbb{N}$, the unions $\mathcal{V}_{k}(S)$ are arithmetical progressions with difference 1 (see [17, Theorem 4.1], 21] for a simpler proof, and also [19]). In [34, unions of sets of lengths are studied for non-principal order in number fields, and in [9], for domains of the form $V+X B[X]$, where $V$ is a discrete valuation domain and $B$ the ring of integers in a finite extension field over the quotient field of $V$. In [2, S.T. Chapman et al. showed that in numerical monoids, generated by arithmetical progressions, all unions are arithmetical progressions. We are going to generalize this result.

Proposition 6.5. Let $S$ be a numerical monoid with $\mathcal{A}(S)=\left\{n_{1}, \ldots, n_{t}\right\}$, where $t \in \mathbb{N}, 1<n_{1}<\ldots<$ $n_{t}$, and $d=\operatorname{gcd}\left(n_{2}-n_{1}, \ldots, n_{t}-n_{t-1}\right)$. Suppose that the Diophantine equations

$$
\left(n_{2}-n_{1}\right) x_{2}+\ldots+\left(n_{t}-n_{1}\right) x_{t}=d n_{1} \quad \text { and } \quad\left(n_{t}-n_{1}\right) y_{1}+\ldots+\left(n_{t}-n_{t-1}\right) y_{t-1}=d n_{t}
$$

have solutions in the non-negative integers. Then there exists an element $a^{*} \in S$ such that $\rho\left(a^{*}\right)=\rho(S)$ and $\mathrm{L}\left(a^{*}\right)$ is an arithmetical progression with difference $d$.

Proof. We proceed in several steps.

1. Let $a \in \mathbb{N}$ be a multiple of $n_{1}$ and of $n_{t}$. Then

$$
z=\frac{a}{n_{1}} \boldsymbol{n}_{\mathbf{1}} \quad \text { and } \quad z^{\prime}=\frac{a}{n_{t}} \boldsymbol{n}_{\boldsymbol{t}}
$$

are factorizations of $a$. Obviously, we have $\min \mathrm{L}(a)=a / n_{t}$, $\max \mathrm{L}(a)=a / n_{1}$ and hence $\rho(a)=n_{1}^{-1} n_{t}$. By Corollary 6.3, 1 it follows that $\rho(a)=\rho(S)$.

2. Since $S$ is finitely generated, Proposition $5.2,2$ and Equation 5.1 imply that $S$ is locally tame with finite set of distances $\Delta(S)$, and $\Delta(S) \neq \emptyset$ because $\rho(S)>1$. Thus [25, Theorem 4.3.6.1] implies that there is an $\bar{a} \in S$ with the following property: for every $b \in S$ we have

$$
\mathrm{L}(\bar{a} b)=y+\left(L^{\prime} \cup L^{*} \cup L^{\prime \prime}\right) \subset y+d \mathbb{Z}
$$

where $y \in \mathbb{Z}, L^{*}$ is an arithmetical progression with difference $d, \min L^{*}=0, L^{\prime} \subset[-\mathrm{t}(S, \mathrm{Z}(\bar{a})),-1]$ and $L^{\prime \prime} \subset \max L^{*}+[1, \mathrm{t}(S, \mathrm{Z}(\bar{a}))]$.

3. Let $\left(\alpha_{2}, \ldots, \alpha_{t}\right) \in \mathbb{N}_{0}^{t-1}$ and $\left(\beta_{1}, \ldots, \beta_{t-1}\right) \in \mathbb{N}_{0}^{t-1}$ be solutions of the given Diophantine equations, and set

$$
\alpha_{1}=\alpha_{2}+\ldots+\alpha_{t} \text { and } \beta_{t}=-\left(\beta_{1}+\ldots+\beta_{t-1}\right) .
$$

Now let $a^{*} \in \mathbb{N}$ be a multiple of $\operatorname{lcm}\left(\bar{a}, n_{1}, n_{t}\right)$ such that

$$
\frac{a^{*}}{n_{1}} \geq \gamma\left(d+\alpha_{1}\right) \quad \text { and } \quad \frac{a^{*}}{n_{t}} \geq \gamma\left|d+\beta_{t}\right| \quad \text { where } \quad \gamma=\left\lceil\frac{\mathrm{t}(S, \mathrm{Z}(\bar{a}))}{d}\right\rceil .
$$

We assert that $a^{*}$ has the required properties. By 1., it follows that

$$
\min \mathrm{L}\left(a^{*}\right)=\frac{a^{*}}{n_{t}}, \max \mathrm{L}\left(a^{*}\right)=\frac{a^{*}}{n_{1}} \quad \text { and } \quad \rho\left(a^{*}\right)=\rho(S) .
$$

We set $a^{*}=\bar{a} b$ with $b \in S$, and write $\mathrm{L}\left(a^{*}\right)$ in the form $\mathrm{L}\left(a^{*}\right)=y+\left(L^{\prime} \cup L^{*} \cup L^{\prime \prime}\right) \subset y+d \mathbb{Z}$ with all properties as in $\mathbf{2}$. (note that such a representation need not be unique).

Let $\nu \in[0, \gamma]$. Then

$$
x_{\nu}=\left(\frac{a^{*}}{n_{1}}-\nu\left(d+\alpha_{1}\right)\right) \boldsymbol{n}_{\mathbf{1}}+\nu \alpha_{2} \boldsymbol{n}_{\mathbf{2}}+\ldots+\nu \alpha_{t} \boldsymbol{n}_{\boldsymbol{t}}
$$

is a factorization of $a^{*}$ of length

$$
\left|x_{\nu}\right|=\frac{a^{*}}{n_{1}}-\nu d-\nu\left(\alpha_{1}-\alpha_{2}-\ldots-\alpha_{t}\right)=\max \mathrm{L}\left(a^{*}\right)-\nu d \in \mathrm{L}\left(a^{*}\right) .
$$


Similarly,

is a factorization of $a^{*}$ of length

$$
y_{\nu}=\left(\frac{a^{*}}{n_{t}}+\nu\left(d+\beta_{t}\right)\right) \boldsymbol{n}_{\boldsymbol{t}}+\nu \beta_{1} \boldsymbol{n}_{\mathbf{1}}+\ldots+\nu \beta_{t-1} \boldsymbol{n}_{t-\mathbf{1}}
$$

$$
\left|y_{\nu}\right|=\frac{a^{*}}{n_{t}}+\nu d+\nu\left(\beta_{1}+\ldots+\beta_{t-1}+\beta_{t}\right)=\min \mathrm{L}\left(a^{*}\right)+\nu d \in \mathrm{L}\left(a^{*}\right) .
$$

This reveals that $\mathrm{L}\left(a^{*}\right)$ starts and ends with arithmetical progressions having difference $d$ and $(\gamma+1)$ elements. Thus it follows that $L^{\prime}$ and $L^{\prime \prime}$ are (possibly empty) arithmetical progressions with difference $d$, and thus $\mathrm{L}\left(a^{*}\right)$ is an arithmetical progression with difference $d$.

Theorem 6.6. Let $S$ be a numerical monoid with $\mathcal{A}(S)=\left\{n_{1}, \ldots, n_{t}\right\}$ where $t \in \mathbb{N}, 1<n_{1}<\ldots<n_{t}$, and $d=\operatorname{gcd}\left(n_{2}-n_{1}, \ldots, n_{t}-n_{t-1}\right)$. Suppose that the Diophantine equations

$$
\left(n_{2}-n_{1}\right) x_{2}+\ldots+\left(n_{t}-n_{1}\right) x_{t}=d n_{1} \quad \text { and } \quad\left(n_{t}-n_{1}\right) y_{1}+\ldots+\left(n_{t}-n_{t-1}\right) y_{t-1}=d n_{t}
$$

have solutions in the non-negative integers. Then there exists a $k^{*} \in \mathbb{N}$ such that $\mathcal{V}_{k}(S)$ is an arithmetical progression with difference $d$ for all $k \geq k^{*}$, and

$$
\lim _{k \rightarrow \infty} \frac{\left|\mathcal{V}_{k}(S)\right|}{k}=\frac{1}{d}\left(\frac{n_{t}}{n_{1}}-\frac{n_{1}}{n_{t}}\right) .
$$

Proof. By Proposition 6.5, all assumptions in [17, Theorem 3.1] are satisfied, and hence this result implies the assertion.

\section{Remarks 6.7.}

1. If $\mathcal{A}(S)$ is an arithmetical progression, then all sets $\mathcal{V}_{k}(S)$ are arithmetical progressions (see [2, Theorem 2.7]). However, in general, we have $k^{*}>2$. Indeed, $S=\langle 4,5,13,14\rangle$ satisfies the assumptions of Theorem 6.6, but since $\mathcal{V}_{2}(S)=\{2,6,7\}$ is not an arithmetical progression, it follows that $k^{*}>2$.

2. Unions of sets of lengths in finitely generated monoids are almost arithmetical progressions (see [19, Theorems 3.5 and 4.2]). But even in a numerical monoid, there may exist infinitely many $k \in \mathbb{N}$, for which these unions are not arithmetical progressions, as the following example shows.

Let $S=\langle 4,10,21\rangle$ and $k \in \mathbb{N}$. Then $d=\operatorname{gcd}(6,11)=1$. We assert that $\mathcal{V}_{k}(S)$ is not an arithmetical progression with difference 1 . We set $S_{k}=\{a \in S \mid k \in \mathrm{L}(a)\}$ and observe that

$$
S_{k}=\left\{a \mathbf{4}+b \mathbf{1 0}+c \mathbf{2 1} \mid a, b, c \in \mathbb{N}_{0} \text { with } a+b+c=k\right\}, \min S_{k}=4 k \quad \text { and } \max S_{k}=21 k .
$$

In particular, we see that $S_{k}=\{4 k, \ldots, 21 k-28,21 k-22,21 k-17,21 k-11,21 k\}$, where the elements are written down in increasing order. The element $21 k$ has a unique factorization of maximal length, namely

$$
\begin{cases}21 t \mathbf{4} & \text { if } k=4 t \\ 21 t \mathbf{4}+\mathbf{2 1} & \text { if } k=4 t+1 \\ (21 t+8) \mathbf{4}+\mathbf{1 0} & \text { if } k=4 t+2 \\ (21 t+8) \mathbf{4}+\mathbf{1 0}+\mathbf{2 1} & \text { if } k=4 t+3\end{cases}
$$

Setting $l=\max \mathrm{L}(21 k)$ we assert that there is no $s \in S_{k}$ with $l-1 \in \mathrm{L}(s)$. If this holds, then $\mathcal{V}_{k}(S)$ is not an arithmetical progression with difference 1 . To verify our assertion we distinguish four cases.

- If $k=4 t$, then $l=21 t$. An element with a factorization of length $21 t-1$ is greater than or equal to $(21 t-1) 4=(21 t) 4-4>21 k-11$, and thus it does not belong to $S_{k}$.

- If $k=4 t+1$, then $l=21 t+1$. Elements having a factorization of length $21 t$ are $(21 t) 4,(21 t-1) 4+$ $10=(21 t) 4+6,(21 t-2) 4+2 \cdot 10=21 t+12,(21 t-1) 4+21=(21 t) 4+17,(21 t-2) 4+10+21=$ $(21 t) 4+23, \ldots$. In this setting the four largest elements of $S_{k}$ are $(21 t) 4+21,(21 t) 4+10,(21 t) 4+4$ and $(21 t) 4-1$. Hence also in this case, there is no element in $S_{k}$ having a factorization of length $l-1$. 
- If $k=4 t+2$, then $l=21 t+9$. The set of elements having a factorization of length $21 t+8$ is $\{(21 t+8) 4=(21 t) 4+32,(21 t+7) 4+10=(21 t) 4+39, \ldots,(21 t+8) 21\}$, and the two largest elements of $S_{k}$ are $(21 t) 4+42$ and $(21 t) 4+31$. Again we see that no element in $S_{k}$ can have a factorization of length $l-1$.

- If $k=4 t+3$, then $l=21 t+10$. Arguing as above one easily checks that no elements in $S_{k}$ have factorizations of length $l-1$.

In view of Theorem 6.6 and the Remarks 6.7 we end this paper with the formulation of the following problem.

Open Problem. Characterize the numerical monoids $S$ for which there exists a $k^{*} \in \mathbb{N}$ such that the unions of sets of lengths $\mathcal{V}_{k}(S)$ are arithmetical progressions for all $k \geq k^{*}$.

\section{REFERENCES}

[1] F. Aguiló-Gost and P.A. García-Sánchez, Factorization and catenary degree in 3-generated numerical semigroups, Electron. Notes Discrete Math. 34 (2009), 157 - 161.

[2] J. Amos, S.T. Chapman, N. Hine, and J. Paixao, Sets of lengths do not characterize numerical monoids, Integers 7 (2007), Paper A50, 8p.

[3] D.F. Anderson, S.T. Chapman, N. Kaplan, and D. Torkornoo, An algorithm to compute w-primality in a numerical monoid, Semigroup Forum, to appear.

[4] V. Barucci, Numerical semigroup algebras, Multiplicative Ideal Theory in Commutative Algebra (J.W. Brewer, S. Glaz, W. Heinzer, and B. Olberding, eds.), Springer, 2006, pp. $39-53$.

[5] — On propinquity of numerical semigroups and one-dimensional local Cohen Macaulay rings, Commutative Algebra and its Applications (M. Fontana, S.-E. Kabbaj, B. Olberding, and I. Swanson, eds.), de Gruyter, 2009, pp. 49 $-60$.

[6] V. Barucci, D.E. Dobbs, and M. Fontana, Maximality Properties in Numerical Semigroups and Applications to OneDimensional Analytically Irreducible Local Domains, vol. 125, Memoirs of the Amer. Math. Soc., 1997.

[7] C. Bowles, S.T. Chapman, N. Kaplan, and D. Reiser, On delta sets of numerical monoids, J. Algebra Appl. 5 (2006), $695-718$.

[8] S.T. Chapman, P.A. García-Sánchez, D. Llena, V. Ponomarenko, and J.C. Rosales, The catenary and tame degree in finitely generated commutative cancellative monoids, Manuscr. Math. 120 (2006), $253-264$.

[9] S.T. Chapman, N. Gonzalez, and S. Pellerin, Sets of lengths in $V+X B[X]$ domains, Arab. J. Sci. Eng., Sect. A, Sci. 26 (2001), $69-82$.

[10] S.T. Chapman, M. Holden, and T. Moore, Full elasticity in atomic monoids and integral domains, Rocky Mt. J. Math. 36 (2006), $1437-1455$.

[11] S.T. Chapman and W.W. Smith, Factorization in Dedekind domains with finite class group, Isr. J. Math. 71 (1990), $65-95$.

[12] E. Contejean and H. Devie, An efficient incremental algorithm for solving systems of linear diophantine equations, Inf. Comput. 113 (1994), $143-172$.

[13] M. Delgado, P.A. García-Sánchez, and J. Morais, "numericalsgps": a gap package on numerical semigroups, ( \protect \vrule widthOpt \protect \href\{http://www.gap-system.org/Packages/numericalsgps.html\}\{http://www.gap-system.org/P

[14] A. Foroutan, Monotone chains of factorizations, Focus on commutative rings research (A. Badawi, ed.), Nova Sci. Publ., New York, 2006, pp. 107 - 130.

[15] A. Foroutan and A. Geroldinger, Monotone chains of factorizations in C-monoids, Arithmetical Properties of Commutative Rings and Monoids, Lect. Notes Pure Appl. Math., vol. 241, Chapman \& Hall/CRC, 2005, pp. 99 - 113.

[16] A. Foroutan and W. Hassler, Chains of factorizations and factorizations with successive lengths, Commun. Algebra 34 (2006), 939 - 972.

[17] M. Freeze and A. Geroldinger, Unions of sets of lengths, Funct. Approximatio, Comment. Math. 39 (2008), $149-162$.

[18] W. Gao and A. Geroldinger, Zero-sum problems in finite abelian groups: a survey, Expo. Math. 24 (2006), $337-369$.

[19] On products of $k$ atoms, Monatsh. Math. 156 (2009), $141-157$.

[20] P.A. García-Sánchez and I. Ojeda, Uniquely presented finitely generated commutative monoids, posted on arxiv.

[21] A. Geroldinger, Additive group theory and non-unique factorizations, Combinatorial Number Theory and Additive Group Theory (A. Geroldinger and I. Ruzsa, eds.), Advanced Courses in Mathematics CRM Barcelona, Birkhäuser, 2009, pp. $1-86$.

[22] A. Geroldinger and D.J. Grynkiewicz, On the arithmetic of Krull monoids with finite Davenport constant, J. Algebra 321 (2009), $1256-1284$. 
[23] A. Geroldinger, D.J. Grynkiewicz, G.J. Schaeffer, and W.A. Schmid, On the arithmetic of Krull monoids with infinite cyclic class group, J. Pure Appl. Algebra, to appear.

[24] A. Geroldinger, D.J. Grynkiewicz, and P. Yuan, On products of $k$ atoms II, manuscript.

[25] A. Geroldinger and F. Halter-Koch, Non-Unique Factorizations. Algebraic, Combinatorial and Analytic Theory, Pure and Applied Mathematics, vol. 278, Chapman \& Hall/CRC, 2006.

[26] A. Geroldinger and W. Hassler, Arithmetic of Mori domains and monoids, J. Algebra 319 (2008), 3419 - 3463.

[27] Local tameness of v-noetherian monoids, J. Pure Appl. Algebra 212 (2008), 1509 - 1524.

[28] A. Geroldinger, W. Hassler, and G. Lettl, On the arithmetic of strongly primary monoids, Semigroup Forum 75 (2007), $567-587$.

[29] A. Geroldinger and F. Kainrath, On the arithmetic of tame monoids with applications to Krull monoids and Mori domains, J. Pure Appl. Algebra, to appear.

[30] P.A. Grillet, Commutative Semigroups, Kluwer Academic Publishers, 2001.

[31] F. Halter-Koch, Ideal Systems. An Introduction to Multiplicative Ideal Theory, Marcel Dekker, 1998.

[32] M. Omidali, The catenary and tame degree of numerical monoids generated by generalized arithmetic sequences, Forum Math., to appear.

[33] I. Peeva and B. Sturmfels, Generic lattice ideals, Amer. Math. Soc. 11 (1998), 363 - 373.

[34] A. Philipp, Arithmetic of non-principal orders in algebraic number fields, manuscript.

[35] - A characterization of arithmetical invariants by the monoid of relations, Semigroup Forum, to appear.

[36] J.C. Rosales and P.A. García-Sánchez, Finitely Generated Commutative Monoids, Nova Science Publishers, 1999

[37] _ Numerical Semigroups, Springer, 2009.

Departamento de Álgebra, Universidad de Granada, Granada 18071, Espana

E-mail address: vblanco@ugr.es, pedro@ugr.es

Institut für Mathematik und Wissenschaftliches Rechnen, Karl-Franzens-Universität Graz, Heinrichstrasse 36, 8010 Graz, Austria

E-mail address: alfred.geroldinger@uni-graz.at 